Sharif University of Technology
Scientia Iranica
Transactions E: Industrial Engineering
hCIENTIA

\title{
Optimizing two multi-echelon inventory systems for perishable products with price and stock dependent demand in supply chain
}

\author{
Z. Dai ${ }^{\mathrm{a}, *}$ K. Gao ${ }^{\mathrm{b}}$, and X. Zheng ${ }^{\mathrm{a}}$ \\ a. School of Management, JiuJiang University, 551 Qianjin East Road, 332005, JiuJiang City, JiangXi Province, China. \\ b. School of Economics, JiuJiang University, 551 Qianjin East Road, 332005, JiuJiang City, JiangXi Province, China.
}

Received 16 April 2019; received in revised form 15 January 2020; accepted 1 June 2020

\author{
KEYWORDS \\ Optimizing; \\ Algorithm; \\ Multi-echelon \\ inventory systems; \\ Perishable products \\ with price and stock \\ dependent demand; \\ Supply chain.
}

\begin{abstract}
Two inventory models for perishable products in the chain are proposed in this paper, one with starting shortages and the other with ending shortages. The demand for perishable products is dependent on price and stock. The supply chain is composed of one manufacturer, one distribution center, and one retailer. The objective of these two models is to maximize the average profit per unit time by determining the optimal replenishment cycle, frequency, and quantity. The property of optimal solutions for two cases of two models is discussed to verify the existence of optimal solutions. Algorithms for searching for optimal solutions are presented. To investigate the effect of parameters on optimal solutions and obtain some management insights, computational experiments with sensitivity analyses are carried out. Finally, conclusions are presented, as well as recommendations for further research.
\end{abstract}

(C) 2022 Sharif University of Technology. All rights reserved.

\section{Introduction}

Inventory cost is an indispensable part of the total cost. The value of inventory is approximately $14 \%$ of the gross domestic product in the United States and inventory control is very important to keep the normal operation of enterprises. Too much or too little stock has a negative impact on the enterprises [1]. High inventory levels increase the responsiveness of enterprises, nevertheless, increase the holding cost of inventory [2]. Low inventory levels reduce the holding inventory cost, but, could cause losses in production

\footnotetext{
*. Corresponding author. Tel.: 0086+18720180263 E-mail addresses: daizhuo1@126.com (Z. Dai); 173814506@qq.com (K. Gao);751758084@qq.com (X. Zheng)
}

doi: $10.24200 /$ sci.2020.53347.3194 and sales. It is necessary to control inventory and maintain an appropriate stock. In other words, inventory decisions should be optimized to minimize the average inventory cost [3] or maximize the average profit per unit time [4]. The purpose of this paper is to maximize the average profit per unit time for two inventory systems, that is, starting with shortages and ending with shortages inventory systems.

Nowadays, to make more profits, many enterprises strengthen cooperation with both upstream and downstream enterprises and form a supply chain by sharing their demand and inventory information [5]. Flexibility and integration in the supply chain play important roles to improve performance [6]. The supply chain includes a set of suppliers, manufacturers, warehouses, distribution centers, and retailers. Merchandises are produced and supplied in the appropriate amounts, to the right locations, at the right times, to minimize system-wide cost while satisfying service level 
requirements, thanks to their coordination. Inventory control is one of the important fields in supply chain management and attracts the attention of scholars and practitioners. They study the optimization problems of two-echelon $[7,8]$ or three-echelon [6] inventory systems in the supply chain. In this paper, two multi-echelon inventory systems will be optimized.

The demand for commodities is affected by many factors. In general, price is the most important factor. Lower prices tend to increase the demand for many commodities, and vice versa $[9,10]$. In other words, demand is the monotone decreasing continuous function of price [11]. In addition to price, many market researchers and sellers of goods have realized that in some retail systems such as supermarkets, the demand for goods is related to the stock in the showrooms or on the shelves. The goods displayed in the showrooms or on the shelves promote the purchasing desire of customers. Therefore, with the increasing quantity of displayed products, the demand for these products will also increase. There are two main reasons for the appearance of stock-dependent demand. One is the selection effect. The other is the advertising effect. In this study, we assumed that price and stock have an impact on demand.

Product perishability is a critical problem for certain enterprises [12]. Perishable products can be classified into two categories. The first category includes products such as blood and drugs with a fixed life that are completely perished at the end of the planning horizon [13]. The second categories contain products such as fresh foods, vegetables, fruits, and flowers with continuous deterioration. For the products of the first categories, there is no residual value at the end. Nevertheless, for the products of the second category, there is some residual value. Thus, these perishable products could be reclaimed by suppliers at a discounted price. In this study, we assumed that the perishable products can be recovered at a discounted price. For the products of the first category, the discounted rate is zero. For the products of the second category, the discounted rate is a positive number less than 1 .

The cost of losses increases as perishable products deteriorate. Hence, the profits of inventory systems are affected by the conditions of perishable products at the time of their deliveries to the customers. If deterioration of perishable products and the cost of losses are not considered, the profits will be exaggerated. Different products have different deterioration rates. For instance, the deterioration speed of fresh food is faster than that of fruit. Some prior investigations assumed that the rate of degradation is constant. According to some studies, the rate of deterioration follows a Weibull distribution. Other studies, such as [14], claim that the rate of deterioration is a function of time. In this paper, the deterioration rate is assumed to be constant.

In summary, this research proposes two multiechelon inventory models, one that ends with a shortage and the other that begins with a shortage. In these two models, perishable products with price and stockdependent demand are considered. Each model, which includes a single manufacturer, distribution center, and retailer is optimized to maximize average profit per unit time by determining not only the optimal replenishment cycle and frequency but also the optimal replenishment quantity. To solve these two models and develop an algorithm, each model is considered in two scenarios, with four propositions offered and proven based on the model analysis.

The rest of this article is organized as follows. Section 2 summarizes the existing research on this topic and highlights the contributions of the present study. Notations and assumptions are presented in Section 3. Sections 5 and 6 put forward two mathematical models and algorithms, respectively. In Section 6, computational experiments are presented. Finally, the findings and recommendations for further research are presented.

\section{Literature review}

\subsection{Optimizing multi-echelon inventory system}

Some scholars investigated the optimization problem of the multi-echelon inventory system. Guerrero et al. [15] put forward a multi-echelon pharmaceutical distribution system, which is composed of external suppliers, center pharmacies, hospital pharmacies, and care units. To minimize the stock-on-hand value, a heuristic algorithm is presented to find near-optimal inventory policies. Tsai and Liu [16] solved a multiechelon inventory problem using a decision support system. This multi-echelon inventory consists of multiple field depots, a warehouse inventory center, and a warehouse repair center. New decision support algorithms are applied in different scenarios. Zhao and Zhao [17] constructed a five-echelon inventory system in two scenarios to analyze the causes of the bullwhip effect. This inventory system includes raw material, supplier, manufacturer, distributor, retailer, and customer. The result shows that the on-hand inventory and the stockout are reversed in the two scenarios. Fichtinger and Yates [18] proposed an optimization approach for multi-echelon supply chain segmentation, which is composed of factories, consolidation centers, and segments. By a real case study, the effectiveness of this approach is demonstrated. Ross et al. [19] used branch-cut and price heuristics to solve a three-echelon location inventory model, which consists of warehouses, distribution centers, retail stores, and donation-only 
centers. The purpose was to determine the location of facilities and to minimize the total annual cost. The effectiveness of the method is validated by numerical examples. Dai et al. [1] presented multi-echelon inventory models with three types of demand in the supply chain. The supply chain is composed of a retailer, a plant, and many middlemen. Computational experiments demonstrated the applicability of the proposed models and the effectiveness of procedures. The impact of parameters on optimal solutions is discussed through sensitivity analyses. Wang et al. [20] considered a multi-echelon inventory system with lateral transshipment and developed an agent-based approach. The efficiency of the proposed method is verified by a twoechelon inventory system. Shen et al. [21] considered a multi-echelon inventory system including a single warehouse and multiple retailers. They tried to find an optimal policy to minimize the average cost of this multi-echelon inventory system. The optimization problem of the multi-echelon inventory system is studied in the above articles. However, the perishability of products is not considered in these articles.

\subsection{Optimizing inventory for perishable products}

Some articles studied the inventory optimization of perishable products. Duan and Liao [22] proposed a new replenishment policy for high perishable products to minimize the outdate rate with a predetermined shortage level. This new replenishment policy is compared with the other two order-up-to policies under two different control modes. The results show this new replenishment policy is the best among all three. The management of perishable product inventories, according to Haijema [23], can be improved by using optimal ordering, issuance, and disposal policies, which can be obtained using stochastic dynamic programming. To verify the validity of the policy proposed in this study, it is compared to a base stock policy, which is commonly used in practice. Under general assumptions, Coelho and Laporte [24] introduced a problem of joint replenishment and delivery of perishable products, which they solved using an exact branch-and-cut algorithm. To evaluate the performance of this algorithm, a set of instances are tested. Liu et al. [25] considered a finite-period inventory model for perishable products and determined the optimal purchasing and inventory retrieval policies to maximize the total expected profit. Computational experiments are performed to study the sensitivity of parameters. Two approximate policies are compared with the proposed policies to demonstrate the value of their model. Kaasgari et al. [26] used Vendor Managed Inventory strategy for perishable products in a two-level supply chain, which is composed of a single vendor and multiple retailers. To minimize the total inventory cost for perishable products, they developed a nonlinear programming model and solved it by a Genetic algorithm and Particle Swarm Optimization (PSO) algorithm. Jaggi et al. [27] studied a twowarehouse inventory system for perishable products with the imperfect quality considering the permissible delay in payments. Numerical examples demonstrated the effectiveness of the proposed model. Teimoury and Kazemi [28] studied a two-stage supply chain model for a single deteriorating product with a constant deteriorating rate to maximize the total profit of the supply chain. Two cases with and without shortage are considered to demonstrate the applicability of the model. The above studies considered the inventory problem of perishable products. However, these studies stipulated that demand is not dependent on stock.

\subsection{Optimizing inventory with stock dependent demand}

Sarkar and Sarkar [29] expanded an inventory model with stock-dependent demand for perishable products to minimize the expected total cost by determining the optimal cycle length. They determined the necessary and sufficient conditions to show the existence and uniqueness of the optimal solution. Finally, some numerical examples are used to demonstrate the application of the proposed model. Panda et al. [30] proposed the selection problem of inventory locations under the assumption that demand is dependent on stock. They developed three mathematical models and provided selection strategies under different conditions. Finally, sensitivity analyses and numerical examples are carried out. Ghiami et al. [31] optimized a two-echelon inventory model with stock-dependent demand. The optimization is done from the perspectives of the wholesaler and retailer. A genetic algorithm is presented to solve this model. A numerical example with sensitivity analysis is carried out. Yang [32] proposed an inventory model with stock-dependent demand and partially backlogged. Moreover, shortages are allowed. This model aims to maximize the total average profit by determining the ending inventory level and the optimal order quantity. Finally, numerical examples and sensitivity analysis are provided. Bhunia et al. [33] developed an inventory model with a perishable product with stock dependent on demand. Two algorithms are applied to solve this model. The impact of different parameters on the optimal solutions is investigated by computational experiments. Lim [34] considered an inventory system with stock dependent on demand and transformed this model into an equivalent programming problem. An optimal inventory replenishment policy is found and validated by a numerical example. The literature cited above examines the inventory optimization problem with stock-dependent demand from various perspectives. 
However, these researches did not take into account the impact of price on demand.

\subsection{Optimizing inventory with price dependent demand}

Panda et al. [4] developed warehouse-inventory models with the assumption that demand is dependent on price and the storage space of retailers is limited. The model with fuzzy parameters aims to maximize the average profit. A genetic algorithm is put forward to solve this model and computational experiments are presented. Maihami and Kamalabadi [35] optimized the joint pricing and inventory model with price and time-dependent demand, shortages allowed, and partially backlogged. The objective of this study was to maximize the total profit. They proved the existence and uniqueness of the solution and proposed an algorithm for finding the optimal solution. Finally, a numerical example is presented to illustrate the algorithm and the solution procedure. Alfares and Ghaithan [11] thought that the demand rate is affected by many factors. One of them is price. Based on this fact, an inventory and pricing model with price-dependent demand, time-varying holding cost, and quantity discounts is developed. A solution method is put forward to determine the optimal solution. A numerical example and sensitivity analysis are carried out. Banerjeea and Agrawal [36] analyzed an inventory model with price-dependent demand. General deterioration distribution and general demand function are considered. Managerial insights are obtained from sensitivity analysis. Jadidi et al. [37] addressed the optimization problem of joint pricing and inventory with price-dependent stochastic demand. Two new mathematical models are formulated. The computational results demonstrated that discounts can increase the profits for the buyer and the supplier and the end customer benefits from a competitive price. San-José et al. [38] analyzed an inventory model with price and time-dependent demand. The objective was to maximize the average profit by using an algorithm. Computational experiments verified the applicability of the model and the effectiveness of the algorithm. Johari et al. [39] put forward a supply chain including a supplier and a retailer whose demand is dependent on price. The results showed that a coordination scheme can improve the profit of the overall supply chain and its member. The above articles enrich the research content of inventory optimization by introducing pricedependent demand. Perishable products, on the other hand, are believed to be entirely perished by the end of the planning horizon and have no residual value, according to these studies.

Based on the above literature, the main contributions of this article are summarized as follows:

- Perishable goods have a residual value at the end of the planning horizon and can be recovered at a discount price. The recovery revenue is composed of one part of the total revenue;

- Two inventory systems are built and optimized, including starting with the shortages inventory system and ending with the shortages inventory system. The analysis of models led to the formulation of eight propositions;

- Price and stock-dependent demand are simultaneously considered. Most previous studies separately considered an inventory system with pricedependent demand or an inventory system with stock-dependent demand;

- Multi-echelon inventory systems are taken into consideration, which is comprised of three subjects: one manufacturer, one distribution center, and one retailer. Previous studies only considered one subject or two subjects.

\section{Notation and assumptions}

\subsection{Notations and indices}

$i \quad$ Index of model $(i=1,2)$

$j \quad$ Index of case $(j=1,2)$

$v \quad$ Index of subcase $(v=1,2,3)$

$t \quad$ Index of time

\section{Parameters}

$p \quad$ Price of unit product

$\theta \quad$ Deterioration rate of perishable product

$\beta \quad$ Impacting coefficient of inventory on demand

$D \quad$ Demand rate of customer

$w \quad$ Recovery price of unit perishable product

$g \quad$ Loss cost of unit perishable product

$h$ Inventory cost of the unit product of retailer

$h_{1} \quad$ Inventory cost of the unit product of distribution center

$h_{2} \quad$ Inventory cost of the unit product of the manufacturer

$s \quad$ Ordering cost of retailer

$s_{1} \quad$ Ordering cost of distribution center

$s_{2} \quad$ The startup cost for production of the manufacturer

c The production cost of unit perishable product

$l \quad$ The maximum quantity of perishable product allowed on the shelf

d Demand related to price 


\section{Decision variables}

$I(t) \quad$ Inventory level at time $t$

\section{Parameters}

$r_{i j(v)} \quad$ Time when the inventory is zero in subcase $v$ of case $j$ of model $i$ (subcase $v$ is optional)

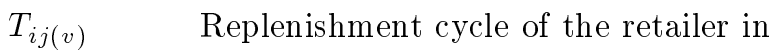
subcase $v$ of case $j$ of model $i$ (subcase $v$ is optional)

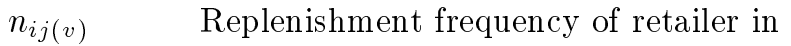
subcase $v$ of case $j$ of model $i$, which is a positive integer number (subcase $v$ is optional)

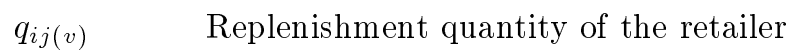
in subcase $v$ of case $j$ of model $i$ (subcase $v$ is optional)

$s q_{i j(v)} \quad$ Shortage quantity of the retailer in subcase $v$ of case $j$ of model $i$ (subcase $v$ is optional)

$R H C_{i j(v)} \quad$ Holding cost of the retailer in subcase $v$ of case $j$ of model $i$ (subcase $v$ is optional)

$R L C_{i j(v)} \quad$ Loss cost of the retailer in subcase $v$ of case $j$ of model $i$ (subcase $v$ is optional)

$D H C_{i j(v)} \quad$ Holding cost of the distribution center in subcase $v$ of case $j$ of model $i$ (subcase $v$ is optional)

$M H C_{i j(v)}$ Holding cost of the manufacturer in subcase $v$ of case $j$ of model $i$ (subcase $v$ is optional)

$S R_{i j(v)} \quad$ Sales revenue in subcase $v$ of case $j$ of model $i$ (subcase $v$ is optional)

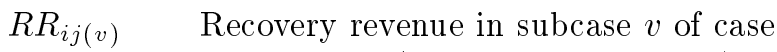
$j$ of model $i$ (subcase $v$ is optional)

$r_{i j(v)}^{*}$

$T_{i j(v)}^{*}$ Optimal time when the inventory is zero in subcase $v$ of case $j$ of model $i$ (subcase $v$ is optional)

Optimal replenishment cycle of retailer in subcase $v$ of case $j$ of model $i$ (subcase $v$ is optional)

$n_{i j(v)}^{*} \quad$ Optimal replenishment frequency of the retailer in subcase $v$ of case $j$ of model $i$, which is a positive integer number (subcase $v$ is optional)

$q_{i j(v)}^{*} \quad$ Optimal replenishment quantity of the retailer in subcase $v$ of case $j$ of model $i$ (subcase $v$ is optional)

$s q_{i j(v)}^{*} \quad$ Optimal shortage quantity of the retailer in subcase $v$ of case $j$ of model $i$ (subcase $v$ is optional)
$A P_{i j(v)}^{*} \quad$ Optimal average profit of the supply chain in subcase $v$ of case $j$ of model $i$ (subcase $v$ is optional)

\subsection{Assumptions}

1. The quantity of perishable products on the shelf is limited.

2. According to Mishra et al. [40] and Chen et al. [41], the demand of customers is affected by the price and stock of a perishable product and inventory level and the demand function can be written as:

$$
D(p, I(t))= \begin{cases}d(p)+\beta I(t) & \text { if } I(t) \geq 0 \\ d(p) & \text { if } I(t)<0\end{cases}
$$

$d(p)$ is a non-negative monotone non-increasing function, that is, $d(p) \geq 0$ and $d^{\prime}(p)<0$.

3. Shortage of the retailer is allowed. The shortage is complete backlogging.

4. According to Teimoury and Kazemi [28] and Pervin et al. [42], the deterioration rate is constant. Deterioration only happens on the shelf of the retailer. The perishable product has a residual value at the end of the planning horizon and is repurchased at a discounted price.

5. The discounted rate is a positive number less than 1 .

\section{Mathematical models}

The multi-echelon inventory system consists of one plant, one distribution center, and one retailer. The average total cost of a multi-echelon inventory system includes the following costs: inventory holding cost, ordering cost, and loss cost of the retailer, ordering cost and holding cost of the distribution center, ordering cost, holding cost, and production cost of the manufacturer. The average revenue is composed of average sales revenue and recovery revenue of residual value. The average profit of the supply chain equals the difference between average revenue and average total cost. This study aims to maximize the average profit of the supply chain by determining $q_{i j}^{*}, r_{i j}^{*}, T_{i j}^{*}$, and $n_{i j}^{*}$. Two models for starting with shortages and ending with shortages are optimized for perishable products with price and stock-dependent demand in the supply chain.

\subsection{Model 1 (ending with shortages)}

\subsubsection{Establishment of model}

The multi-echelon inventory model ending with shortages is shown in Figure 1. The purpose of this model is to maximize the average profit of the supply chain.

The inventory cost of retailer

As we can see from Figure 1, the replenishment arrives 


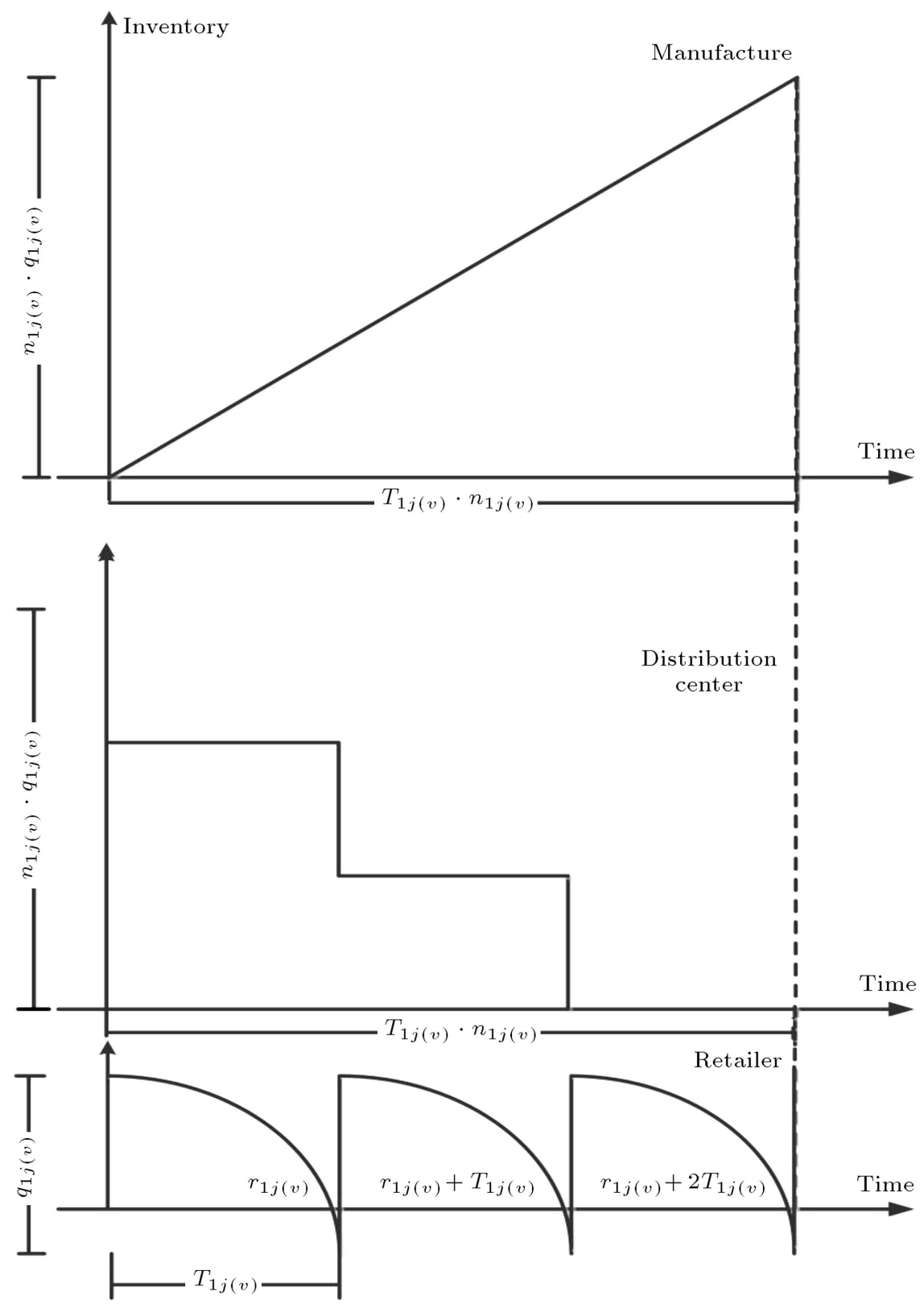

Figure 1. Inventory system ending with shortage.

in retailer at time 0 . The inventory of retailer is decreased to zero at time $r_{1 j(v)}$ due to the demand of customers and the deterioration of perishable products. The demand after time $r_{1 j(v)}$ is backlogging. Based on the above description, the inventory at time $t$ could be formulated by the following equation:

$$
\begin{aligned}
& \frac{d I(t)}{d t} \\
& = \begin{cases}-d(p)-\beta I(t)-\theta I(t) & 0 \leq t \leq r_{1 j(v)} \\
-d(p) & r_{1 j(v)} \leq t \leq T_{1 j(v)}\end{cases}
\end{aligned}
$$

We solve the above equation with the boundary condition $I\left(r_{1 j(v)}\right)=0$. The solution is given by:

$$
\begin{aligned}
& I(t) \\
& = \begin{cases}\frac{d(p)}{\beta+\theta}\left(\mathrm{e}^{(\beta+\theta)\left(r_{1 j(v)}-t\right)}-1\right) & 0 \leq t \leq r_{1 j(v)} \\
d(p)\left(r_{1 j(v)}-T_{1 j(v)}\right) & r_{1 j(v)} \leq t \leq T_{1 j(v)}(2)\end{cases}
\end{aligned}
$$

The holding cost of the retailer from time 0 to time $r_{1 j(v)}$ is calculated by:

$$
R H C_{i j(v)}=h \int_{0}^{r_{1 j(v)}} I(t) d t
$$




$$
\begin{aligned}
& =h \int_{0}^{r_{1 j(v)}} \frac{d(p)}{\beta+\theta}\left(e^{(\beta+\theta)\left(r_{1 j(v)}-t\right)}-1\right) d t \\
& =\frac{h d(p)}{(\beta+\theta)^{2}}\left(e^{(\beta+\theta) r_{1 j(v)}}-1\right)-\frac{h d(p) r_{1 j(v)}}{(\beta+\theta)} .
\end{aligned}
$$

The loss cost of the retailer from time 0 to time $r_{1 j(v)}$ is calculated by:

$$
\begin{aligned}
R L C_{1 j(v)}= & g \int_{0}^{r_{1 j(v)}} \theta I(t) d t \\
= & \theta g\left(\frac{d(p)}{(\beta+\theta)^{2}}\left(e^{(\beta+\theta) r_{1 j(v)}}-1\right)\right. \\
& \left.-\frac{d(p)}{(\beta+\theta)} r_{1 j(v)}\right) .
\end{aligned}
$$

The ordering cost of the retailer from time 0 to time $T_{1 j(v)}$ is $s$. The shortage quantity from $r_{1 j(v)}$ to time $T_{1 j(v)}$ is:

$$
s q_{1 j(v)}=d(p)\left(T_{1 j(v)}-r_{1 j(v)}\right) .
$$

The order quantity of the retailer from time 0 to time $T_{1 j(v)}$ is given by:

$$
\begin{aligned}
q_{1 j(v)}= & I(0)+s q_{1 j(v)}=\frac{d(p)}{\beta+\theta}\left(e^{(\beta+\theta) r_{1 j(v)}}-1\right) \\
& +d(p)\left(T_{1 j(v)}-r_{1 j(v)}\right) .
\end{aligned}
$$

If $r_{1 j(v)}^{*}$ and $T_{1 j(v)}^{*}$ are determined, the optimal order quantity $q_{1 j(v)}^{*}$ is written as:

$$
\begin{aligned}
q_{1 j(v)}^{*}= & I(0)+s q_{1 j(v)}=\frac{d(p)}{\beta+\theta}\left(e^{\left.(\beta+\theta) r_{1 j(v)}^{*}-1\right)}\right. \\
& +d(p)\left(T_{1 j(v)}^{*}-r_{1 j(v)}^{*}\right) .
\end{aligned}
$$

The inventory cost of the distribution center and manufacturer

The ordering cost of a distribution center in $n_{1 j(v)} T_{1 j(v)}$ is $s_{1}$. As shown in Figure 1, the holding cost of the distribution center in $n_{1 j(v)} T_{1 j(v)}$ is calculated by:

$$
D H C_{1 j(v)}=h_{1} T_{1 j(v)} q_{1 j(v)} \frac{n_{1 j(v)}\left(n_{1 j(v)}-1\right)}{2} .
$$

The startup cost of the manufacturer in $n_{1 j(v)} T_{1 j(v)}$ is $s_{2}$. The production cost of the manufacturer in $n_{1 j(v)} T_{1 j(v)}$ is $c n_{1 j(v)} q_{1 j(v)}$. As we can see from Figure 1, the holding cost of the manufacturer in $n_{1 j(v)} T_{1 j(v)}$ is:

$$
M H C_{1 j(v)}=\left(n_{1 j(v)}\right)^{2} q_{1 j(v)} T_{1 j(v)} h_{2} / 2 .
$$

The revenue of supply chain

The sales revenue of the supply chain from 0 to $T_{1 j(v)}$ is written as:

$$
\begin{aligned}
S R_{1 j(v)}= & \int_{0}^{r_{1 j(v)}}(d(p)+\beta I(t)) d t \\
& +p d(p)\left(T_{1 j(v)}-r_{1 j(v)}\right) \\
= & p\left(d(p) r_{1 j(v)}+\frac{d(p) \beta}{(\beta+\theta)^{2}}\left(e^{(\beta+\theta) r_{1 j(v)}}-1\right)\right. \\
& \left.-\frac{d(p) \beta}{\beta+\theta} r_{1 j(v)}+d(p)\left(T_{1 j(v)}-r_{1 j(v)}\right)\right) .
\end{aligned}
$$

The recovery revenue of the supply chain from 0 to $T_{1 j(v)}$ is given by:

$$
\begin{aligned}
R R_{1 j(v)}= & w \int_{0}^{r_{1 j(v)}} \frac{d(p) \theta}{\beta+\theta}\left(e^{(\beta+\theta)\left(r_{1 j(v)}-t\right)}-1\right) d t \\
= & \frac{w d(p) \theta}{(\beta+\theta)^{2}}\left(e^{\left.(\beta+\theta) r_{1 j(v)}-1\right)}\right. \\
& -\frac{w d(p) \theta r_{1 j(v)}}{\beta+\theta} .
\end{aligned}
$$

Based on the above calculation, the average profit of the supply chain is formulated as follows:

$$
\begin{aligned}
A P_{1 j(v)} & \left(n_{1 j(v),} r_{1 j(v)}, T_{1 j(v)}\right)=p\left(d(p) r_{1 j(v)}\right. \\
& +\frac{d(p) \beta}{(\beta+\theta)^{2}}\left(e^{(\beta+\theta) r_{1 j(v)}}-1\right) \\
& \left.-\frac{d(p) \beta}{\beta+\theta} r_{1 j(v)}+d(p)\left(T_{1 j(v)}-r_{1 j(v)}\right)\right) / T_{1 j(v)} \\
& +\left(\frac{w d(p) \theta}{(\beta+\theta)^{2}}\left(e^{(\beta+\theta) r_{1 j}(v)}-1\right)\right. \\
& \left.-\frac{w d(p) \theta r_{1 j(v)}}{\beta+\theta}\right) / T_{1 j(v)} \\
& -\left(\frac{h d(p)}{(\beta+\theta)^{2}}\left(e^{(\beta+\theta) r_{1 j(v)}}-1\right)\right. \\
& \left.-\frac{h d(p) r_{1 j(v)}}{(\beta+\theta)}\right) / T_{1 j(v)} \\
& -\theta g\left(\frac{d(p)}{(\beta+\theta)^{2}}\left(e^{(\beta+\theta) r_{1 j(v)}}-1\right)\right. \\
& \left.-\frac{d(p)}{(\beta+\theta)} r_{1 j(v)}\right) / T_{1 j(v)}-s / T_{1 j(v)} \\
- & s_{1} / n_{1 j(v)} / T_{1 j(v)}-h_{1} T_{1 j(v)} \\
q_{1 j(v)} & \frac{n_{1 j(v)}\left(n_{1 j(v)}-1\right)}{2} / n_{1 j(v)} / T_{1 j(v)}
\end{aligned}
$$




$$
\begin{aligned}
& -\left(n_{1 j(v)}\right)^{2} q_{1 j(v)} T_{1 j(v)} h_{2} / 2 / n_{1 j(v)} / T_{1 j(v)} \\
& -s_{2} / n_{1 j(v)} / T_{1 j(v)} \\
& -c n_{1 j(v)} q_{1 j(v)} / n_{1 j(v)} / T_{1 j(v)} .
\end{aligned}
$$

The objective of this model is to maximize $A P_{1 j(v)}$ $\left(n_{1 j(v)}, r_{1 j(v)}, T_{1 j(v)}\right)$ with the storage space limitation of shelf by determining $n_{1 j(v)}, r_{1 j(v)}$, and $T_{1 j(v)}$.

\subsubsection{Analysis of model}

This subsection analyzes the model in two cases, demonstrates the uniqueness of the solutions, and provides the solutions.

Based on Eq. (12), we can see $A P_{1 j(v)}$ is the function of variables $n_{1 j(v)}, r_{1 j(v)}$, and $T_{1 j(v)}$. We discuss the impact of $n_{1 j(v)}$ on $A P_{1 j(v)}$ under the assumption that $r_{1 j(v)}$ and $T_{1 j(v)}$ are fixed. Taking the first-order and second-order derivative of $A P_{1 j(v)}$ with respect to $n_{1 j(v)}$, we obtain:

$$
\begin{gathered}
\frac{\partial A P_{1 j(v)}\left(n_{1 j(v),} r_{1 j(v)}, T_{1 j(v)}\right)}{\partial n}=\frac{s_{1}}{T_{1 j(v)} n_{1 j(v)}^{2}} \\
-\frac{h_{1} q_{1 j(v)}}{2}-\frac{q_{1 j(v)} h_{2}}{2}+\frac{s_{2}}{T_{1 j(v)} n_{1 j(v)}^{2}}, \\
\frac{\partial^{2} A P_{1 j(v)}\left(n_{1 j(v)}, r_{1 j(v)}, T_{1 j(v)}\right)}{\partial n_{1 j(v)}^{2}}= \\
-\frac{2 s_{1}}{T_{1 j(v)} n_{1 j(v)}^{3}}-\frac{2 s_{2}}{T_{1 j(v)} n_{1 j(v)}^{3}}<0 .
\end{gathered}
$$

Eq. (14) implies $A P_{1 j(v)}$ is strictly concave. Therefore, Eq. $(13)=0$ has at most one solution. Then, we discuss the property of $A P_{1 j(v)}\left(n_{1 j(v)}, r_{1 j(v)}, T_{1 j(v)}\right)$ with respect to $r_{1 j(v)}$ and $T_{1 j(v)}$ if $n_{1 j(v)}^{*}$ is given. Plugging Eq. (6) into Eq. (12) and taking the firstorder of $A P_{1 j(v)}$ with respect to $r_{1 j(v)}$, we obtain:

$$
\begin{gathered}
\frac{\partial A P_{1 j(v)}\left(n_{1 j(v)}^{*}, r_{1 j(v)}, T_{1 j(v)}\right)}{\partial r_{1 j(v)}}=\left(d(p) e^{(\beta+\theta) r_{1 j(v)}}\right. \\
-d(p))\left(\frac{p \beta+w \theta-h-\theta g-c(\beta+\theta)}{T_{1 j(v)}(\beta+\theta)}\right. \\
\left.-\frac{h_{1}\left(n_{1 j(v)}^{*}-1\right)+n_{1 j(v)}^{*} h_{2}}{2}\right) .
\end{gathered}
$$

Based on the value of $p \beta+w \theta-h-\theta g-c(\beta+\theta)$, two cases are discussed for Model 1.

Case 1: $\quad p \beta+w \theta-h-\theta g-c(\beta+\theta) \leq 0$.

Proposition 1. For a given $n_{11}^{*}$, if $p \beta+w \theta-h-$ $\theta g-c(\beta+\theta) \leq 0$, the optimal solution of Eq. (12) is $\left(n_{11}^{*}, 0, T_{11}^{*}\right)$ where $T_{11}^{*}$ could be obtained by the following equation:

$$
\begin{aligned}
& \frac{\partial A P_{11}\left(n_{11}^{*}, 0, T_{11}\right)}{\partial T_{11}}=\frac{s}{T_{11}{ }^{2}}+\frac{s_{1}}{n_{11}^{*} T_{11}{ }^{2}} \\
& -\frac{h_{1}\left(n_{11}^{*}-1\right) d\left(p_{11}\right)}{2}-\frac{n_{11}^{*} d\left(p_{11}\right) h_{2}}{2} \\
& +\frac{s_{2}}{n_{11}^{*} T_{11}{ }^{2}}=0 .
\end{aligned}
$$

The proof of proposition 1 is in Appendix A.

Case 2: $\quad p \beta+w \theta-h-\theta g-c(\beta+\theta)>0$.

There are three subcases to discuss.

Case 2.1. $\quad \frac{p \beta+w \theta-h-\theta g-c(\beta+\theta)}{T_{121}(\beta+\theta)}-\frac{h_{1}\left(n_{121}-1\right)+n_{121} h_{2}}{2}<0$.

Proposition 2. For a given $n_{121}^{*}$, if:

$$
\frac{p \beta+w \theta-h-\theta g-c(\beta+\theta)}{T_{121}(\beta+\theta)}-\frac{h_{1}\left(n_{121}^{*}-1\right)+n_{121}^{*} h_{2}}{2}<0,
$$

the optimal solution of Eq. (12) is $\left(n_{121}^{*}, 0, T_{121}^{*}\right)$ where $T_{121}^{*}$ could be obtained by the following equation. Otherwise, $\left(n_{121}^{*}, 0, T_{121}^{*}\right)$ is not a feasible solution.

$$
\begin{gathered}
\frac{\partial A P_{121}\left(n_{121}^{*}, 0, T_{121}\right)}{\partial T_{121}}=\frac{s}{T_{121}{ }^{2}}+\frac{s_{1}}{n_{121}^{*} T_{121}{ }^{2}} \\
-\frac{h_{1}\left(n_{121}^{*}-1\right) d(p)}{2}-\frac{n_{121}^{*} d(p) h_{2}}{2} \\
+\frac{s_{2}}{n_{121}^{*} T_{121}{ }^{2}}=0 .
\end{gathered}
$$

The proof of Proposition 2 is in Appendix B.

Case 2.2 $\frac{p \beta+w \theta-h-\theta g-c(\beta+\theta)}{T_{122}(\beta+\theta)}-\frac{h_{1}\left(n_{122}-1\right)+n_{122} h_{2}}{2}>0$.

Proposition 3. For a given $n_{122}^{*}$, if:

$\frac{p \beta+w \theta-h-\theta g-c(\beta+\theta)}{T_{122}(\beta+\theta)}-\frac{h_{1}\left(n_{122}^{*}-1\right)+n_{122}^{*} h_{2}}{2}>0$,

the optimal solution of Eq. (12) is $\left(n_{122}^{*}, T_{122}^{*}, T_{122}^{*}\right)$ where $T_{122}^{*}$ could be obtained by the following equation. Otherwise, $\left(n_{122}^{*}, T_{122}^{*}, T_{122}^{*}\right)$ is not a feasible solution.

$$
T_{122}^{*}=\ln \left(\frac{l(\beta+\theta)}{d(p)}+1\right) /(\beta+\theta) \text {. }
$$

The proof of Proposition 3 is in Appendix C.

Case 2.3 $\frac{p \beta+w \theta-h-\theta g-c(\beta+\theta)}{T_{123}(\beta+\theta)}-\frac{h_{1}\left(n_{123}-1\right)+n_{123} h_{2}}{2}=0$.

Proposition 4. If: 
$\frac{p \beta+w \theta-h-\theta g-c(\beta+\theta)}{T_{123}(\beta+\theta)}-\frac{h_{1}\left(n_{123}-1\right)+n_{123} h_{2}}{2}=0$,

there is no optimal value for $A P_{123}\left(n_{123}, r_{123}, T_{123}\right)$. The proof of Proposition 4 is in Appendix D.

\subsection{Model 2 (starting with shortages)}

\subsubsection{Establishment of model}

The inventory cost of retailer

The multi-echelon inventory model starting with shortages is shown in Figure 2.

The replenishment arrives at the retailer at time $r_{2 j(v)}$. The inventory of retailer is decreased to zero at time $T_{2 j(v)}$ due to the demand of customers and the deterioration of perishable products. The demand from time 0 to time $r_{2 j(v)}$ is complete backlogging. Based on the above description, the inventory at time $t$ could be formulated by the following equation:

$$
\begin{aligned}
& \frac{d I(t)}{d t}= \\
& \begin{cases}-d(p) & 0 \leq t \leq r_{2 j(v)} \\
-d(p)-\beta I(t)-\theta I(t) & r_{2 j(v)} \leq t \leq T_{2 j(v)}\end{cases}
\end{aligned}
$$

The above equation is solved with the boundary condition $I\left(r_{2 j(v)}\right)=0$. The solution is written as:

$$
\begin{aligned}
& I(t)= \\
& \begin{cases}-d(p) t & 0 \leq t \leq r_{2 j(v)} \\
\frac{d(p)}{\beta+\theta}\left(e^{(\beta+\theta)\left(T_{2 j(v)}-t\right)}-1\right) & r_{2 j(v)} \leq t \leq T_{2 j(v)}(19)\end{cases}
\end{aligned}
$$

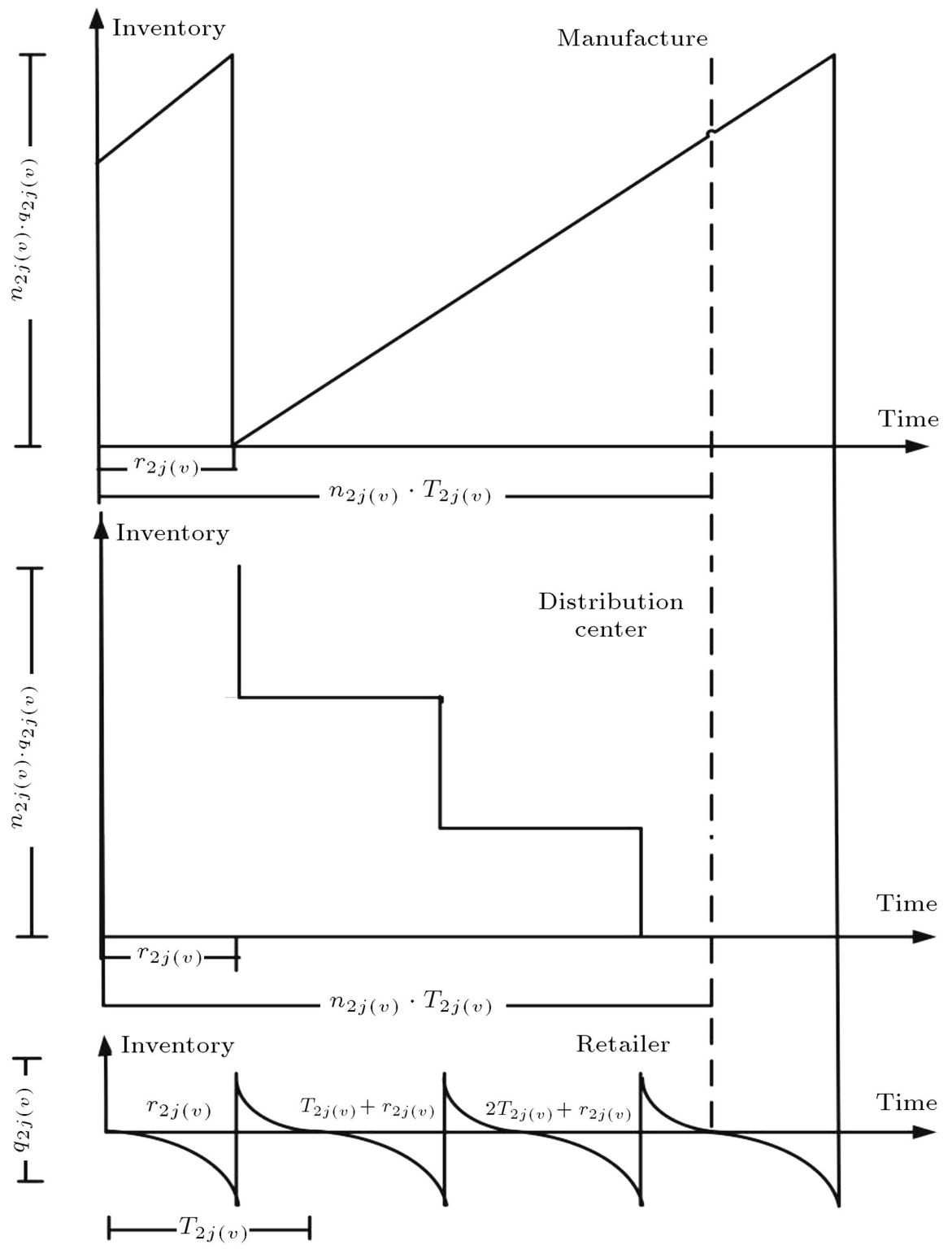

Figure 2. Inventory system starting with shortage. 
The holding cost of the retailer from time $r_{2 j(v)}$ to time $T_{2 j(v)}$ is given by:

$$
\begin{aligned}
R H C_{2 j(v)}= & h \int_{r_{2 j(v)}}^{T_{2 j(v)}} I(t) d t \\
= & h \int_{r_{2 j(v)}}^{T_{2 j(v)}} \frac{d(p)}{\beta+\theta}\left(e^{(\beta+\theta)\left(T_{\left.2 j(v)-r_{2 j(v)}\right)}-1\right) d t}\right. \\
= & \frac{h d(p)}{(\beta+\theta)^{2}}\left(e^{(\beta+\theta)\left(T_{2 j(v)}-r_{2 j(v)}\right)}-1\right) \\
& -\frac{h d(p)\left(T_{2 j(v)}-r_{2 j(v)}\right)}{(\beta+\theta)} .
\end{aligned}
$$

The loss cost of the retailer from time $r_{2 j(v)}$ to time $T_{2 j(v)}$ is calculated by:

$$
\begin{aligned}
R L C_{2 j(v)}= & g \int_{r_{2 j(v)}}^{T_{2 j(v)}} \theta I(t) d t=\theta g\left(\frac{d(p)}{(\beta+\theta)^{2}}\right. \\
& \left(e^{(\beta+\theta)\left(T_{2 j(v)}-r_{2 j(v)}\right)}-1\right) \\
& \left.-\frac{d(p)}{(\beta+\theta)}\left(T_{2 j(v)}-r_{2 j(v)}\right)\right) .
\end{aligned}
$$

The ordering cost of the retailer from time $r_{2 j(v)}$ to time $T_{2 j(v)}$ is $s$. The shortage quantity from time 0 to time $r_{2 j(v)}$ is:

$$
s q_{2 j(v)}=d(p) r_{2 j(v)} .
$$

The order quantity of retailer from time 0 to time $T_{2 j(v)}$ is calculated by:

$$
\begin{aligned}
q_{2 j(v)}= & I(0)+s q_{2 j(v)}=\frac{d(p)}{\beta+\theta}\left(e^{(\beta+\theta)\left(T_{\left.2 j(v)-r_{2 j(v)}\right)}-1\right)}\right. \\
& +d(p) r_{2 j(v)} .
\end{aligned}
$$

If $r_{2 j(v)}^{*}$ and $T_{2 j(v)}^{*}$ are determined, the optimal order quantity $q_{2 j(v)}^{*}$ is given by:

$$
\begin{aligned}
q_{2 j(v)}^{*}= & I(0)+s q_{2 j(v)}=\frac{d(p)}{\beta+\theta} \\
& \left(e^{(\beta+\theta)\left(T_{2 j(v)}^{*}-r_{2 j(v)}^{*}\right)}-1\right)+d(p) r_{2 j(v)}^{*} .
\end{aligned}
$$

The inventory cost of distribution center and manufacturer

The ordering cost of the distribution center in $n_{2 j(v)} T_{2 j(v)}$ is $s_{1}$. As we can see from Figure 2, the holding cost of the distribution center in $n_{2 j(v)} T_{2 j(v)}$ is written as:

$$
\begin{aligned}
D H C_{2 j(v)}= & h_{1}\left(0 \cdot r_{2 j(v)}+\left(n_{2 j(v)}-1\right) q_{2 j(v)} T_{2 j(v)}\right. \\
& +\left(n_{2 j(v)}-2\right) \\
& q_{2 j(v)} T_{2 j(v)}+\cdots q_{2 j(v)} T_{2 j(v)}
\end{aligned}
$$

$$
\begin{aligned}
& \left.+0 \cdot\left(T_{2 j(v)}-r_{2 j(v)}\right)\right)=h_{1} T_{2 j(v)} q_{2 j(v)} \\
& \frac{n_{2 j(v)}\left(n_{2 j(v)}-1\right)}{2} .
\end{aligned}
$$

The startup cost of the manufacturer in $n_{2 j(v)} T_{2 j(v)}$ is $s_{2}$. The production cost of the manufacturer in $n_{2 j(v)} T_{2 j(v)}$ is $c n_{2 j(v)} q_{2 j(v)}$. The holding cost of the manufacturer in $n_{2 j(v)} T_{2 j(v)}$ is:

$$
\begin{gathered}
M H C_{2 j(v)}=h_{2}\left(2 n_{2 j(v)} q_{2 j(v)}-r_{2 j(v)} q_{2 j(v)} / T_{2 j(v)}\right) \\
r_{2 j(v)} / 2+h_{2}\left(n_{2 j(v)} T_{2 j(v)}-r_{2 j(v)}\right) \\
\left(n_{2 j(v)} q_{2 j(v)}-r_{2 j(v)} q_{2 j(v)} / T_{2 j(v)}\right) / 2 \\
=\left(n_{2 j(v)}\right)^{2} q_{2 j(v)} T_{2 j(v)} h_{2} / 2
\end{gathered}
$$

The revenue of supply chain

The sales revenue of the supply chain from 0 to $T_{2 j(v)}$ is given by:

$$
\begin{aligned}
S R_{2 j(v)}= & p \int_{r_{2 j(v)}}^{T_{2 j(v)}}(d(p)+\beta I(t)) d t \\
& +p d(p) r_{2 j(v)}=p d(p) T_{2 j(v)} \\
& -\frac{p d(p) \beta}{(\beta+\theta)^{2}}\left(1-e^{(\beta+\theta)\left(T_{2 j(v)}-r_{2 j(v)}\right)}\right) \\
& -\frac{p d(p) \beta}{(\beta+\theta)}\left(T_{2 j(v)}-r_{2 j(v)}\right) .
\end{aligned}
$$

The recovery revenue of the supply chain from 0 to $T_{2 j(v)}$ is calculated by:

$$
\begin{aligned}
R R_{2 j(v)} & =w \int_{r_{2 j(v)}}^{T_{2 j(v)}} \frac{d(p) \theta}{\beta+\theta}\left(e^{(\beta+\theta)\left(T_{2 j(v)}-r_{2 j(v)}\right)}-1\right) d t \\
& =\frac{w d(p) \theta}{(\beta+\theta)^{2}}\left(e^{(\beta+\theta)\left(T_{2 j(v)}-r_{2 j(v)}\right)}-1\right) \\
& -\frac{w d(p) \theta}{\beta+\theta}\left(T_{2 j(v)}-r_{2 j(v)}\right) .
\end{aligned}
$$

Based on the above calculation, the average profit of the supply chain is given as follows:

$$
\begin{aligned}
A P_{2 j(v)}( & \left.n_{2 j(v),} r_{2 j(v)}, T_{2 j(v)}\right)=p_{2 j(v)}\left(d(p) T_{2 j(v)}\right. \\
& +\frac{d(p) \beta}{(\beta+\theta)^{2}}\left(e^{(\beta+\theta)\left(T_{2 j(v)}-r_{2 j(v)}\right)}-1\right) \\
& \left.-\frac{d(p) \beta}{\beta+\theta}\left(T_{2 j(v)}-r_{2 j(v)}\right)\right) / T_{2 j(v)} \\
& +\left(\frac{w d(p) \theta}{(\beta+\theta)^{2}}\left(e^{(\beta+\theta)\left(T_{2 j(v)}-r_{2 j(v)}\right)}-1\right)\right.
\end{aligned}
$$




$$
\begin{aligned}
& \left.-\frac{w d(p) \theta\left(T_{2 j(v)}-r_{2 j(v)}\right)}{\beta+\theta}\right) / T_{2 j(v)} \\
& -\left(\frac{h d(p)}{(\beta+\theta)^{2}}\left(e^{(\beta+\theta)\left(T_{2 j(v)}-r_{2 j(v)}\right)}-1\right)\right. \\
& \left.-\frac{h d(p)\left(T_{2 j(v)}-r_{2 j(v)}\right)}{(\beta+\theta)}\right) / T_{2 j(v)} \\
& -\theta g\left(\frac{d(p)}{(\beta+\theta)^{2}}\left(e^{(\beta+\theta)\left(T_{2 j(v)}-r_{2 j(v)}\right)}-1\right)\right. \\
& \left.-\frac{d(p)\left(T_{2 j(v)}-r_{2 j(v)}\right)}{(\beta+\theta)}\right) / T_{2 j(v)}-s / T_{2 j(v)} \\
& -s_{1} / n_{2 j(v)} / T_{2 j(v)}-h_{1} T_{2 j(v)} q_{2 j(v)} \\
& \frac{n_{2 j(v)}\left(n_{2 j(v)}-1\right)}{2} / n_{2 j(v)} / T_{2 j(v)} \\
& -\left(n_{2 j(v)}\right)^{2} q_{2 j(v)} T_{2 j(v)} h_{2} / 2 / n_{2 j(v)} / T_{2 j(v)} \\
& -s_{2} / n_{2 j(v)} / T_{2 j(v)}-c n_{2 j(v)} q_{2 j(v)} / n_{2 j(v)} / T_{2 j(v)} .
\end{aligned}
$$

The purpose of this model is to maximize $A P_{2 j(v)}$ $\left(n_{2 j(v)}, r_{2 j(v)}, T_{2 j(v)}\right)$ with the space limitation of shelf by determining $n_{2 j(v)}, r_{2 j(v)}$, and $T_{2 j(v)}$.

\subsubsection{Analysis of model}

In this subsection, the model is analyzed in two cases and the solutions and their properties are provided.

Based on Eq. (29), we can see $A P_{2 j(v)}$ is the function of variables $n_{2 j(v)}, r_{2 j(v)}$, and $T_{2 j(v)}$. We study the impact of $n_{2 j(v)}$ on $A P_{2 j(v)}$ under the assumption that $r_{2 j(v)}$ and $T_{2 j(v)}$ are fixed. We take the first-order and second-order derivatives of $A P_{2 j(v)}$ with respect to $n_{2 j(v)}$.

$$
\begin{aligned}
& \frac{\partial A P_{2 j(v)}\left(n_{2 j(v),} r_{2 j(v)}, T_{2 j(v)}\right)}{\partial n_{2 j(v)}}=\frac{s_{1}}{T_{2 j(v)} n_{2 j(v)}^{2}} \\
& -\frac{h_{1} q_{2 j(v)}}{2}-\frac{q_{2 j(v)} h_{2}}{2}+\frac{s_{2}}{T_{2 j(v)} n_{2 j(v)}^{2}}, \\
& \frac{\partial^{2} A P_{2 j(v)}\left(n_{2 j(v)}, r_{2 j(v)}, T_{2 j(v)}\right)}{\partial n_{2 j(v)}^{2}}=-\frac{2 s_{1}}{T_{2 j(v)} n_{2 j(v)}^{3}} \\
& -\frac{2 s_{2}}{T_{2 j(v)} n_{2 j(v)}^{3}}<0 .
\end{aligned}
$$

Eq. (31) indicates Eq. (30) $=0$ has at most one solution. Then, we discuss the property of $A P_{2 j(v)}$ $\left(n_{2 j(v)}, r_{2 j(v)}, T_{2 j(v)}\right)$ with respect to $r_{2 j(v)}$ and $T_{2 j(v)}$ if $n_{2 j(v)}^{*}$ is given. We plug Eq. (23) into Eq. (29) and take the first-order of $A P_{2 j(v)}$ with respect to $r_{2 j(v)}$.

$$
\begin{gathered}
\frac{\partial A P_{2 j(v)}\left(n_{2 j(v)}^{*}, r_{2 j(v)}, T_{2 j(v)}\right)}{\partial r_{2 j(v)}}= \\
\left(-d(p) e^{(\beta+\theta)\left(T_{2 j(v)}-r_{2 j(v)}\right)}+d(p)\right) \\
\left(\frac{p \beta+w \theta-h-\theta g-c(\beta+\theta)}{T_{2 j(v)}(\beta+\theta)}\right. \\
\left.-\frac{h_{1}\left(n_{2 j(v)}^{*}-1\right)+n_{2 j(v)}^{*} h_{2}}{2}\right) .
\end{gathered}
$$

Based on the value of $p \beta+w \theta-h-\theta g-c(\beta+\theta)$, two cases are discussed for Model 2.

Case 1: $\quad p \beta+w \theta-h-\theta g-c(\beta+\theta) \leq 0$.

Proposition 5. For a given $n_{21}^{*}$, if $p \beta+w \theta-h-$ $\theta g-c(\beta+\theta) \leq 0$, the optimal solution of Eq. (29) is $\left(n_{21}^{*}, T_{21}^{*}, T_{21}^{*}\right)$ where $T_{21}^{*}$ could be obtained by the following equation:

$$
\begin{gathered}
\frac{\partial A P_{21}\left(n_{21}^{*}, T_{21}, T_{21}\right)}{\partial T_{21}}=\frac{s}{T_{21}{ }^{2}}+\frac{s_{1}}{n_{21}^{*} T_{21}{ }^{2}} \\
-\frac{h_{1}\left(n_{21}^{*}-1\right) d(p)}{2}-\frac{n_{21}^{*} d(p) h_{2}}{2} \\
+\frac{s_{2}}{n_{21}^{*} T_{21}{ }^{2}}=0 .
\end{gathered}
$$

The proof of Proposition 5 is in Appendix E.

Case 2: $\quad p \beta+w \theta-h-\theta g-c(\beta+\theta)>0$. There are three subcases to discuss.

Case 2.1: $\quad \frac{p \beta+w \theta-h-\theta g-c(\beta+\theta)}{T_{221}(\beta+\theta)}-\frac{h_{1}\left(n_{221}-1\right)+n_{221} h_{2}}{2}<0$.

Proposition 6. For a given $n_{221}^{*}$, if:

$$
\frac{p \beta+w \theta-h-\theta g-c(\beta+\theta)}{T_{221}(\beta+\theta)}-\frac{h_{1}\left(n_{221}^{*}-1\right)+n_{221}^{*} h_{2}}{2}<0,
$$

the optimal solution of Eq. (29) is $\left(n_{221}^{*}, T_{221}^{*}, T_{221}^{*}\right)$ where $T_{221}^{*}$ could be solved by the following equation, otherwise $\left(n_{221}^{*}, T_{221}^{*}, T_{221}^{*}\right)$ is not a feasible solution.

$$
\begin{gathered}
\frac{\partial A P_{221}\left(n_{221}^{*}, T_{221}, T_{221}\right)}{\partial T_{221}}=\frac{s}{T_{221}{ }^{2}}+\frac{s_{1}}{n_{221}^{*} T_{221}{ }^{2}} \\
-\frac{h_{1}\left(n_{221}^{*}-1\right) d(p)}{2}-\frac{n_{221}^{*} d(p) h_{2}}{2} \\
+\frac{s_{2}}{n_{221}^{*} T_{221}{ }^{2}}=0 .
\end{gathered}
$$

The proof of Proposition 6 is in Appendix F. 
Case 2.2: $\quad \frac{p \beta+w \theta-h-\theta g-c(\beta+\theta)}{T_{222}(\beta+\theta)}-\frac{h_{1}\left(n_{222}-1\right)+n_{222} h_{2}}{2}>0$

Proposition 7. For a given $n_{222}^{*}$, if:

$$
\frac{p \beta+w \theta-h-\theta g-c(\beta+\theta)}{T_{222}(\beta+\theta)}-\frac{h_{1}\left(n_{222}^{*}-1\right)+n_{222}^{*} h_{2}}{2}>0,
$$

the optimal solution of Eq. (29) is $\left(n_{222}^{*}, 0, T_{222}^{*}\right)$ where $T_{222}^{*}$ could be solved by the following equation. Otherwise, $\left(n_{222}^{*}, 0, T_{222}^{*}\right)$ is not a feasible solution.

$$
T_{222}^{*}=\ln \left(\frac{l(\beta+\theta)}{d\left(p_{222}\right)}+1\right) /(\beta+\theta) .
$$

The proof of Proposition 7 is in Appendix G.

Case 2.3 $\frac{p \beta+w \theta-h-\theta g-c(\beta+\theta)}{T_{223}(\beta+\theta)}-\frac{h_{1}\left(n_{223}-1\right)+n_{223} h_{2}}{2}=0$.

Proposition 8. If:

$$
\frac{p \beta+w \theta-h-\theta g-c(\beta+\theta)}{T_{223}(\beta+\theta)}-\frac{h_{1}\left(n_{223}-1\right)+n_{223} h_{2}}{2}=0,
$$

there is no optimal value for $A P_{223}\left(n_{223}, r_{223}, T_{223}\right)$. The proof of Proposition 8 is in Appendix $\mathrm{H}$.

The above two models can be simplified to other models. First, if $\theta=0$, these two models are simplified to the models for non-perishable products. Second, if $w=0$, recovering for perishable products will not be considered. Third, if $\beta=0$, demand is not dependent on stock. Fourth, if $d(p)=0$, demand will not be affected by price. Therefore, these two models include at least four simplified models. In other words, simplified models that appear in other studies are special cases of the proposed models in this paper.

\section{Algorithms}

\subsection{Algorithm for Model 1}

Based on the analysis of Subsection 4.1.2, we can use the following algorithm to solve Model 1:

Step 1. Set the value for parameters;

Step 2. If $p \beta+w \theta-h-\theta g-c(\beta+\theta) \leq 0$, go to algorithm 1-1;

Step 3. If $p \beta+w \theta-h-\theta g-c(\beta+\theta)>0$, go to Algorithm 1-2.

\section{Algorithm 1-1}

Step 1. Set $r_{11}^{*}=0$ and $n_{11}^{*}=1$;

Step 2. Obtain $T_{11, n_{11}^{*}}^{*}$ by solving Eq. (16) and plug $T_{11, n_{11}^{*}}^{*}$ and $r_{11}^{*}$ into Eq. ( 7$)$ to obtain $q_{11, n_{11}^{*}}^{*}$;

Step 3. Plug $q_{11, n_{11}^{*}}^{*}, n_{11}^{*}, r_{11}^{*}$, and $T_{11, n_{11}^{*}}^{*}$ into Eq. (12) to obtain $A P_{11}^{*}\left(1, r_{11}^{*}, T_{11, n_{11}^{*}}^{*}\right)$;

Step 4. $n_{11}^{*}=1+n_{11}^{*}$;
Step 5. Obtain $T_{11, n_{11}^{*}}^{*}$ by solving Eq. (16) and plug $T_{11, n_{11}^{*}}^{*}$ and $r_{11}^{*}$ into Eq. (7) to obtain $q_{11, n_{11}^{*}}^{*}$;

Step 6. Plug $q_{11, n_{11}^{*}}^{*} n_{11}^{*}, \quad r_{11}^{*}$, and $T_{11, n_{11}^{*}}^{*}$ into Eq. (12) to obtain $A P_{11}^{*}\left(n_{11}^{*}, r_{11}^{*}, T_{11, n_{11}^{*}}^{*}\right)$;

Step 7. If $A P_{11}^{*}\left(n_{11}^{*}, r_{11}^{*}, T_{11, n_{11}^{*}}^{*}\right)>A P_{11}^{*}\left(n_{11}^{*}-1\right.$, $\left.r_{11}^{*}, T_{11, n_{11}^{*}-1}^{*}\right)$, go to Step 4 , otherwise go to Step 8;

Step 8. Output the optimal solution $\left(n_{11}^{*}-1, r_{11}^{*}\right.$, $\left.T_{11, n_{11}^{*}-1}^{*}\right)$.

\section{Algorithm 1-2}

Step 1. Set $r_{121}^{*}=0, n_{12}^{*}=1$;

Step 2. Obtain $T_{121, n_{12}^{*}}^{*}$ by solving Eq. (17-1) and plug $T_{121, n_{12}^{*}}^{*}$ and $r_{121}^{*}$ into Eq. (7) to obtain $q_{121, n_{12}^{*}}^{*}$

Step 3. If $\frac{p \beta+w \theta-h-\theta g-c(\beta+\theta)}{T_{121, n_{12}^{*}}^{*}(\beta+\theta)}-\frac{h_{1}\left(n_{12}^{*}-1\right)+n_{12}^{*} h_{2}}{2}<0$, plug $q_{121, n_{12}^{*}}^{*}, n_{12}^{*}, r_{121}^{*}$, and $T_{121, n_{12}^{*}}^{*}$ into Eq. (12) to obtain $A P_{121}^{*}\left(n_{12}^{*}, r_{121}^{*}, T_{121, n_{12}^{*}}^{*}\right)$, otherwise let $A P_{121}^{*}\left(n_{12}^{*}, r_{121}^{*}, T_{121, n_{12}^{*}}^{*}\right)=$ -10000 ;

Step 4. Obtain $T_{122, n_{12}^{*}}^{*}$ by solving Eq. (17-2) and set $r_{122, n_{12}^{*}}^{*}=T_{122, n_{12}^{*}}^{*}$. Then plug $T_{122, n_{12}^{*}}^{*}$ and $r_{122, n_{12}^{*}}^{*}$ into Eq. (7) to obtain $q_{122, n_{12}^{*}}^{*}$;

Step 5. If $\frac{p \beta+w \theta-h-\theta g-c(\beta+\theta)}{T_{122, n_{12}^{*}}^{*}(\beta+\theta)}-\frac{h_{1}\left(n_{12}^{*}-1\right)+n_{12}^{*} h_{2}}{2}>$ 0 , plug $q_{122, n_{12}^{*}}^{*}, n_{12}^{*}, r_{122, n_{12}^{*}}^{*}$ and $T_{122, n_{12}^{*}}^{*}$ into Eq. (12) to obtain $A P_{122}^{*}\left(n_{122}^{*}, r_{122, n_{12}^{*}}^{*}\right.$, $\left.T_{122, n_{12}^{*}}^{*}\right)$, otherwise, let $A P_{122}^{*}\left(n_{12}^{*}, r_{122, n_{12}^{*}}^{*}\right.$, $\left.T_{122, n_{12}^{*}}^{*}\right)=-10000$;

Step 6. $A P_{12}^{*}\left(n_{12}^{*}, r_{12, n_{12}^{*}}^{*}, T_{12, n_{12}^{*}}^{*}\right)=\max \left\{A P_{121}^{*}\left(n_{12}^{*}\right.\right.$, $\left.\left.r_{121}^{*}, T_{121, n_{12}^{*}}^{*}\right), A P_{122}^{*}\left(n_{12}^{*}, r_{122, n_{12}^{*}}^{*}, T_{122, n_{12}^{*}}^{*}\right)\right\}$;

Step 7. Set $n_{12}^{*}=1+n_{12}^{*}$;

Step 8. Obtain $T_{121, n_{12}^{*}}^{*}$ by solving Eq. (17-1) and plug $T_{121, n_{12}^{*}}^{*}$ and $r_{121}^{*}$ into Eq. (7) to obtain $q_{121, n_{12}^{*}}^{*}$

Step 9. If $\frac{p \beta+w \theta-h-\theta g-c(\beta+\theta)}{T_{121, n_{12}^{*}}^{*}(\beta+\theta)}-\frac{h_{1}\left(n_{12}^{*}-1\right)+n_{12}^{*} h_{2}}{2}<0$, plug $q_{121, n_{12}^{*}}^{*}, n_{12}^{*}, r_{121}^{*}$, and $T_{121, n_{12}^{*}}^{*}$ into Eq. (12) to obtain $A P_{121}^{*}\left(n_{12}^{*}, r_{121}^{*}, T_{121, n_{12}^{*}}^{*}\right)$, otherwise, let $A P_{121}^{*}\left(n_{12}^{*}, r_{121}^{*}, T_{121, n_{12}^{*}}^{*}\right)=$ $-10000$

Step 10. Obtain $T_{122, n_{12}^{*}}^{*}$ by solving Eq. (17-2) and set $r_{122, n_{12}^{*}}^{*}=T_{122, n_{12}^{*}}^{*}$. Then plug $T_{122, n_{12}^{*}}^{*}$ and $r_{122, n_{12}^{*}}^{*}$ into Eq. ( 7$)$ to obtain $q_{122, n_{12}^{*}}^{*}$;

Step 11. If $\frac{p \beta+w \theta-h-\theta g-c(\beta+\theta)}{T_{122, n_{12}^{*}}^{*}(\beta+\theta)}-\frac{h_{1}\left(n_{12}^{*}-1\right)+n_{12}^{*} h_{2}}{2}>0$, plug $q_{122, n_{12}^{*}}^{*}, n_{12}^{*}, r_{122, n_{12}^{*}}^{*}$ and $T_{122, n_{12}^{*}}^{*}$ into Eq. (12) to obtain $A P_{122}^{*}\left(n_{12}^{*}, r_{122, n_{12}^{*}}^{*}\right.$, $\left.T_{122, n_{12}^{*}}^{*}\right)$, otherwise, let $A P_{122}^{*}\left(n_{12}^{*}, r_{122, n_{12}^{*}}^{*}\right.$, $\left.T_{122, n_{12}^{*}}^{*}\right)=-10000$; 
Step 12. $A P_{12}^{*}\left(n_{12}^{*}, r_{12, n_{12}^{*}}^{*}, T_{12, n_{12}^{*}}^{*}\right)=$ $\max \left\{A P_{121}^{*}\left(n_{12}^{*}, r_{121}^{*}, T_{121, n_{12}^{*}}^{*}\right)\right.$, $\left.A P_{122}^{*}\left(n_{12}^{*}, r_{122, n_{12}^{*}}^{*}, T_{122, n_{12}^{*}}^{*}\right)\right\}$

Step 13. If $A P_{12}^{*}\left(n_{12}^{*}, r_{12, n_{12}^{*}}^{*}, T_{12, n_{12}^{*}}^{*}\right)>A P_{12}^{*}\left(n_{12}^{*}-1\right.$, $r_{12, n_{12}^{*}-1}^{*}, T_{12, n_{12}^{*}-1}^{*}$, go to Step 7 , otherwise go to Step 14;

Step 14. Output the optimal solution $\left(n_{12}^{*}-1\right.$, $\left.r_{12, n_{12}^{*}-1}^{*}, T_{12, n_{12}^{*}-1}^{*}\right)$.

\subsection{Algorithm for Model 2}

Based on the analysis of Subsection 4.2.2, we can use the following algorithm to solve Model 2.

Step 1. Set the value for parameters;

Step 2. If $p \beta+w \theta-h-\theta g-c(\beta+\theta) \leq 0$, go to Algorithm 2-1;

Step 3. If $p \beta+w \theta-h-\theta g-c(\beta+\theta)>0$, go to Algorithm 2-2.

\section{Algorithm 2-1}

Step 1. Set $n_{21}^{*}=1$;

Step 2. Obtain $T_{21, n_{21}^{*}}^{*}$ by solving Eq. (33), set $r_{21, n_{21}^{*}}^{*}=T_{21, n_{21}^{*}}^{*}$, and plug $T_{21, n_{21}^{*}}^{*}$ and $r_{21, n_{21}^{*}}^{*}$ into Eq. (24) to obtain $q_{21, n_{21}^{*}}^{*}$;

Step 3. Plug $q_{21, n_{21}^{*}}^{*}, n_{21}^{*}, r_{21, n_{21}^{*}}^{*}$, and $T_{21, n_{21}^{*}}^{*}$ into Eq. (29) to obtain $A P_{21}^{*}\left(n_{21}^{*}, r_{21, n_{21}^{*}}^{*}, T_{21, n_{21}^{*}}^{*}\right)$;

Step 4. $n_{21}^{*}=1+n_{21}^{*}$;

Step 5. Obtain $T_{21, n_{21}^{*}}^{*}$ by solving Eq. (33) and plug $T_{21, n_{21}^{*}}^{*}$ and $r_{21, n_{21}^{*}}^{*}$ into Eq. (24) to obtain $q_{21, n_{21}^{*}}^{*}$

Step 6. Plug $q_{21, n_{21}^{*}}^{*} n_{21}^{*}, r_{21, n_{21}^{*}}^{*}$, and $T_{21, n_{21}^{*}}^{*}$ into Eq. (29) to obtain $A P_{21}^{*}\left(n_{21}^{*}, r_{21, n_{21}^{*}}^{*}, T_{21, n_{21}^{*}}^{*}\right)$;

Step 7. If $A P_{21}^{*}\left(n_{21}^{*}, r_{21, n_{21}^{*}}^{*}, T_{21, n_{21}^{*}}^{*}\right)>A P_{21}^{*}\left(n_{21}^{*}-1\right.$, $\left.r_{21, n_{21}^{*}-1}^{*}, T_{21, n_{21}^{*}-1}^{*}\right)$, go to Step 4 , otherwise, go to Step 8;

Step 8. Output the optimal solution $\left(n_{21}^{*}-1\right.$, $\left.r_{21, n_{21}^{*}-1}^{*}, T_{21, n_{21}^{*}-1}^{*}\right)$.

\section{Algorithm 2-2}

Step 1. Set $n_{22}^{*}=1$;

Step 2. Obtain $T_{221, n_{22}^{*}}^{*}$ by solving Eq. (34-1), set $r_{221, n_{22}^{*}}^{*}=T_{221, n_{22}^{*}}^{*}$, and plug $T_{221, n_{22}^{*}}^{*}$ and $r_{221, n_{22}^{*}}^{*}$ into Eq. (24) to obtain $q_{221, n_{22}^{*}}^{*}$;

Step 3. If $\frac{p \beta+w \theta-h-\theta g-c(\beta+\theta)}{T_{221, n_{22}^{*}}^{*}(\beta+\theta)}-\frac{h_{1}\left(n_{22}^{*}-1\right)+n_{22}^{*} h_{2}}{2}<$ 0 , plug $q_{221, n_{22}^{*}}^{*}, n_{22}^{*}, r_{221, n_{22}^{*}}^{*}$ and $T_{221, n_{22}^{*}}^{*}$ into Eq. (29) to obtain $A P_{221}^{*}\left(n_{22}^{*}, r_{221, n_{22}^{*}}^{*}\right.$, $\left.T_{221, n_{22}^{*}}^{*}\right)$, otherwise, let $A P_{221}^{*}\left(n_{22}^{*}, r_{221, n_{22}^{*}}^{*}\right.$, $\left.T_{221, n_{22}^{*}}^{*}\right)=-10000$;

Step 4. Obtain $T_{222, n_{22}^{*}}^{*}$ by solving Eq. (34-2) and set $r_{222}^{*}=0$. Then plug $T_{222, n_{22}^{*}}^{*}$ and $r_{222}^{*}$ into Eq. (24) to obtain $q_{222, n_{22}^{*}}^{*}$;
Step 5. If $\frac{p \beta+w \theta-h-\theta g-c(\beta+\theta)}{T_{222, n_{22}^{*}}^{*}(\beta+\theta)}-\frac{h_{1}\left(n_{22}^{*}-1\right)+n_{22}^{*} h_{2}}{2}>0$, plug $q_{222, n_{22}^{*}}^{*}, n_{22}^{*}, r_{222}^{*}$ and $T_{222, n_{22}^{*}}^{*}$ into Eq. (29) to obtain $A P_{222}^{*}\left(n_{22}^{*}, r_{222}^{*}, T_{222, n_{22}^{*}}^{*}\right)$, otherwise, let $A P_{222}^{*}\left(n_{22}^{*}, r_{222}^{*}, T_{222, n_{22}^{*}}^{*}\right)=$ -10000 ;

Step 6. $A P_{22}^{*}\left(n_{22}^{*}, r_{22,1}^{*}, T_{22,1}^{*}\right)=\max \left\{A P_{221}^{*}\left(n_{22}^{*}\right.\right.$, $\left.\left.r_{221, n_{22}^{*}}^{*}, T_{221, n_{22}^{*}}^{*}\right), A P_{222}^{*}\left(n_{22}^{*}, r_{222}^{*}, T_{222, n_{22}^{*}}^{*}\right)\right\}$;

Step 7. Set $n_{22}^{*}=1+n_{22}^{*}$;

Step 8. Obtain $T_{221, n_{22}^{*}}^{*}$ by solving Eq. (34), set $r_{221, n_{22}^{*}}^{*}=T_{221, n_{22}^{*}}^{*}$ and plug $T_{221, n_{22}^{*}}^{*}$ and $r_{221, n_{22}^{*}}^{*}$ into Eq. (24) to obtain $q_{221, n_{22}^{*}}^{*}$;

Step 9. If $\frac{p \beta+w \theta-h-\theta g-c(\beta+\theta)}{T_{221, n_{22}^{*}}^{*}(\beta+\theta)} \frac{h_{1}\left(n_{22}^{*}-1\right)+n_{22}^{*} h_{2}}{2}<0$, plug $q_{221, n_{22}^{*}}^{*}, n_{22}^{*}, r_{221, n_{22}^{*}}^{*}$ and $T_{221, n_{22}^{*}}^{*}$ into Eq. (29) to obtain $A P_{221}^{*}\left(n_{22}^{*}, r_{221, n_{22}^{*}}^{*}\right.$, $\left.T_{221, n_{22}^{*}}^{*}\right)$, otherwise, let $A P_{221}^{*}\left(n_{22}^{*}, r_{221, n_{22}^{*}}^{*}\right.$, $\left.T_{221, n_{22}^{*}}^{*}\right)=-10000$;

Step 10. Obtain $T_{222, n_{22}^{*}}^{*}$ by solving Eq. (34-2) and set $r_{222}^{*}=0$. Then plug $T_{222, n_{22}^{*}}^{*}$ and $r_{222}^{*}$ into Eq. (24) to obtain $q_{222, n_{22}^{*}}^{*}$;

Step 11. If $\frac{p \beta+w \theta-h-\theta g-c(\beta+\theta)}{T_{222, n_{22}^{*}}^{*}(\beta+\theta)}-\frac{h_{1}\left(n_{22}^{*}-1\right)+n_{22}^{*} h_{2}}{2}>$ 0 , plug $q_{222, n_{22}^{*}}^{*}, n_{22}^{*}, r_{222}^{*}$ and $T_{222, n_{22}^{*}}^{*}$ into Eq. (29) to obtain $A P_{222}^{*}\left(n_{22}^{*}, r_{222}^{*}, T_{222, n_{22}^{*}}^{*}\right)$, otherwise, let $A P_{222}^{*}\left(n_{22}^{*}, r_{222}^{*}, T_{222, n_{22}^{*}}^{*}\right)=$ -10000 ;

Step 12. $A P_{22}^{*}\left(n_{22}^{*}, r_{22, n_{22}^{*}}^{*}, T_{22, n_{22}^{*}}^{*}\right)=\max \left\{A P_{221}^{*}\left(n_{22}^{*}\right.\right.$, $\left.\left.r_{221, n_{22}^{*}}^{*}, T_{221, n_{22}^{*}}^{*}\right), A P_{222}^{*}\left(n_{22}^{*}, r_{222}^{*}, T_{222, n_{22}^{*}}^{*}\right)\right\}$;

Step 13. If $A P_{22}^{*}\left(n_{22}^{*}, r_{22, n_{22}^{*}}^{*}, T_{22, n_{22}^{*}}^{*}\right)>A P_{22}^{*}\left(n_{22}^{*}-\right.$ $\left.1, r_{22, n_{22}^{*}-1}^{*}, T_{22, n_{22}^{*}-1}^{*}\right)$, go to Step 7 , otherwise, go to Step 14;

Step 14. Output the optimal solution $\left(n_{22}^{*}-1\right.$, $\left.r_{22, n-1}^{*}, T_{22, n-1}^{*}\right)$.

\section{Computational experiments}

In this section, two multi-echelon inventory systems for perishable products are solved using the algorithm proposed in Section 5. Sensitivity analyses for two cases of two models are performed. All programs are coded in MATLAB and run on a PC with a Pentium (R) Processor (2 GHz) and $2 \mathrm{~GB}$ of memory.

6.1. Numerical example for Case 1 of Model 1 The parameters for Case 1 of model 1 are shown in Table 1 . The demand function is $d(p)=9500-500 p$.

Table 1. Parameters for Case 1 of Model 1.

\begin{tabular}{ccccccccccccc}
$\boldsymbol{p}$ & $\boldsymbol{\beta}$ & $\boldsymbol{\theta}$ & $\boldsymbol{w}$ & $\boldsymbol{h}$ & $\boldsymbol{h}_{\mathbf{1}}$ & $\boldsymbol{h}_{\mathbf{2}}$ & $\boldsymbol{g}$ & $\boldsymbol{f}$ & $\boldsymbol{s}$ & $\boldsymbol{s}_{\mathbf{1}}$ & $\boldsymbol{s}_{\mathbf{2}}$ & $\boldsymbol{l}$ \\
\hline 10 & 0.6 & 0.3 & 1 & 3 & 1 & 5 & 6 & 5 & 5 & 3 & 3 & 150 \\
\hline
\end{tabular}


According to Algorithm 1-1 presented in Subsection 5.1 , we can obtain the following optimal results:

$$
\begin{aligned}
& r_{11}^{*}=0, \quad T_{11}^{*}=0.0313, \quad n_{11}^{*}=1, \\
& q_{11}^{*}=140.71, \quad A P_{11}^{*}=21796.44 .
\end{aligned}
$$

$r_{11}^{*}=0$ indicates that the holding cost, loss cost of the retailer, and recovery revenue of the supply chain is zero. $n_{11}^{*}=1$ means that the holding cost of the distribution center is zero.

Sensitivity analysis is used to investigate the impact of parameters on optimal solutions. All parameters take four values. $p, \beta, \theta$, and $w$ reduce $10 \%$ each time. $h, h_{1}, h_{2}, g, c, s, s_{1}$, and $s_{2}$ increase $10 \%$ each time. $l$ increases 50 each time. The results of sensitivity analysis are presented in Table 2.

As we can see from Table 2, none of the parameters have any effect on $n_{11}^{*}$ and $r_{11}^{*}$. $p$ has a positive correlation with $T_{11}^{*}$ and $A P_{11}^{*}$. Nevertheless, $p$ has a negative correlation with $q_{11}^{*} \cdot \beta, \theta, w, h, h_{1}, g$, and $l$ have no effect on $T_{11}^{*}, q_{11}^{*}$ and $A P_{11}^{*}$. $h_{2}$ has a negative relationship with $T_{11}^{*}, q_{11}^{*}$, and $A P_{11}^{*}$. $c$ has no effect on $T_{11}^{*}$ and $q_{11}^{*}$. And $c$ has a negative correlation with $A P_{11}^{*} \cdot s, s_{1}$, and $s_{2}$ are positively related to $T_{11}^{*}$ and $T_{11}^{*}$ and $q_{11}^{*}$ are negatively related to $A P_{11}^{*}$.

Next, we analyze the reasons for the impact of the parameters on $A P_{11}^{*}$. The reason that $p$ has a positive impact on $A P_{11}^{*}$ is that sales revenue increases with increasing $p$. Since the holding cost of the retailer, loss cost of the retailer, recovery revenue of supply chain, and the holding cost of the distribution center are zero, $\beta, \theta, w, h, h_{1}$, and $g$ do not affect $A P_{11}^{*}$. With increasing $h_{2}, c, s, s_{1}$, and $s_{2}$, the ordering cost and holding cost increased which led to the decrease of $A P_{11}^{*}$. Finally, $l$ does not work as the inventory of the retailer is zero. Therefore, $l$ does not affect $A P_{11}^{*}$.

6.2. Numerical example for Case 2 of Model 1 The parameters for Case 2 of Model 1 are shown in Table 3. The demand function is $d(p)=9500-500 p$. According to the Algorithm 1-2 given in Subsection 5.1, the following optimal results can be obtained:

$$
\begin{aligned}
& r_{12}^{*}=0.0186, \quad T_{12}^{*}=0.0186, \quad n_{12}^{*}=8, \\
& q_{12}^{*}=150, \quad A P_{12}^{*}=18518.27 .
\end{aligned}
$$

$r_{11}^{*}=T_{12}^{*}=0$ indicates that no shortage happens. $q_{12}^{*}=150$ implies that the space of the shelf is fully used.

Sensitivity analysis is performed to investigate the impact of parameters on optimal solutions. Similar to Case 1, all parameters take four values. $p, \beta, \theta$, and $w$ increase $10 \%$ each time. $h, h_{1}, h_{2}, g, c, s, s_{1}$, and

\begin{tabular}{|c|c|c|c|c|c|c|}
\hline Parameter & Value & $n_{11}^{*}$ & $r_{11}^{*}$ & $T_{11}^{*}$ & $q_{11}^{*}$ & $A P_{11}^{*}$ \\
\hline \multirow[t]{4}{*}{$p$} & 10 & 1 & 0 & 0.0313 & 140.71 & 21796.4 \\
\hline & 9 & 1 & 0 & 0.0297 & 148.32 & 19258.4 \\
\hline & 8 & 1 & 0 & 0.0283 & 155.56 & 15722.2 \\
\hline & 7 & 1 & 0 & 0.0271 & 162.48 & 11187.6 \\
\hline \multirow[t]{4}{*}{$\beta$} & 0.6 & 1 & 0 & 0.0313 & 140.71 & 21796.4 \\
\hline & 0.54 & 1 & 0 & 0.0313 & 140.71 & 21796.4 \\
\hline & 0.48 & 1 & 0 & 0.0313 & 140.71 & 21796.4 \\
\hline & 0.42 & 1 & 0 & 0.0313 & 140.71 & 21796.4 \\
\hline \multirow[t]{4}{*}{$\theta$} & 0.3 & 1 & 0 & 0.0313 & 140.71 & 21796.4 \\
\hline & 0.27 & 1 & 0 & 0.0313 & 140.71 & 21796.4 \\
\hline & 0.24 & 1 & 0 & 0.0313 & 140.71 & 21796.4 \\
\hline & 0.21 & 1 & 0 & 0.0313 & 140.71 & 21796.4 \\
\hline \multirow[t]{4}{*}{$w$} & 1 & 1 & 0 & 0.0313 & 140.71 & 21796.4 \\
\hline & 0.9 & 1 & 0 & 0.0313 & 140.71 & 21796.4 \\
\hline & 0.8 & 1 & 0 & 0.0313 & 140.71 & 21796.4 \\
\hline & 0.7 & 1 & 0 & 0.0313 & 140.71 & 21796.4 \\
\hline \multirow[t]{4}{*}{$h$} & 3 & 1 & 0 & 0.0313 & 140.71 & 21796.4 \\
\hline & 3.3 & 1 & 0 & 0.0313 & 140.71 & 21796.4 \\
\hline & 3.6 & 1 & 0 & 0.0313 & 140.71 & 21796.4 \\
\hline & 3.9 & 1 & 0 & 0.0313 & 140.71 & 21796.4 \\
\hline \multirow[t]{4}{*}{$h_{1}$} & 1 & 1 & 0 & 0.0313 & 140.71 & 21796.4 \\
\hline & 1.1 & 1 & 0 & 0.0313 & 140.71 & 21796.4 \\
\hline & 1.2 & 1 & 0 & 0.0313 & 140.71 & 21796.4 \\
\hline & 1.3 & 1 & 0 & 0.0313 & 140.71 & 21796.4 \\
\hline \multirow[t]{4}{*}{$h_{2}$} & 5 & 1 & 0 & 0.0313 & 140.71 & 21796.4 \\
\hline & 5.5 & 1 & 0 & 0.0298 & 134.16 & 21762.1 \\
\hline & 6 & 1 & 0 & 0.0285 & 128.45 & 21729.3 \\
\hline & 6.5 & 1 & 0 & 0.0274 & 123.41 & 21697.8 \\
\hline \multirow[t]{4}{*}{$g$} & 6 & 1 & 0 & 0.0313 & 140.71 & 21796.4 \\
\hline & 6.6 & 1 & 0 & 0.0313 & 140.71 & 21796.4 \\
\hline & 7.2 & 1 & 0 & 0.0313 & 140.71 & 21796.4 \\
\hline & 7.8 & 1 & 0 & 0.0313 & 140.71 & 21796.4 \\
\hline \multirow[t]{4}{*}{$c$} & 5 & 1 & 0 & 0.0313 & 140.71 & 21796.4 \\
\hline & 5.5 & 1 & 0 & 0.0313 & 140.71 & 19546.4 \\
\hline & 6 & 1 & 0 & 0.0313 & 140.71 & 17296.4 \\
\hline & 6.5 & 1 & 0 & 0.0313 & 140.71 & 15046.4 \\
\hline \multirow[t]{4}{*}{$s$} & 5 & 1 & 0 & 0.0313 & 140.71 & 21796.4 \\
\hline & 5.5 & 1 & 0 & 0.0320 & 143.87 & 21780.6 \\
\hline & 6 & 1 & 0 & 0.0327 & 146.97 & 21765.2 \\
\hline & 6.5 & 1 & 0 & 0.0333 & 150.00 & 21750.0 \\
\hline \multirow[t]{4}{*}{$s_{1}$} & 3 & 1 & 0 & 0.0313 & 140.71 & 21796.4 \\
\hline & 3.3 & 1 & 0 & 0.0317 & 142.62 & 21786.9 \\
\hline & 3.6 & 1 & 0 & 0.0321 & 144.50 & 21777.5 \\
\hline & 3.9 & 1 & 0 & 0.0325 & 146.36 & 21768.2 \\
\hline \multirow[t]{4}{*}{$s_{2}$} & 3 & 1 & 0 & 0.0313 & 140.71 & 21796.4 \\
\hline & 3.3 & 1 & 0 & 0.0317 & 142.62 & 21786.9 \\
\hline & 3.6 & 1 & 0 & 0.0321 & 144.50 & 21777.5 \\
\hline & 3.9 & 1 & 0 & 0.0325 & 146.36 & 21768.2 \\
\hline \multirow[t]{4}{*}{$l$} & 150 & 1 & 0 & 0.0313 & 140.71 & 21796.4 \\
\hline & 200 & 1 & 0 & 0.0313 & 140.71 & 21796.4 \\
\hline & 250 & 1 & 0 & 0.0313 & 140.71 & 21796.4 \\
\hline & 300 & 1 & 0 & 0.0313 & 140.71 & 21796.4 \\
\hline
\end{tabular}
$s_{2}$ decrease $10 \%$ each time. $l$ increases $50 \%$ each time. The results are shown in Table 4 .
Table 2. Sensitivity analysis for Case 1 of Model 1 . 
Table 3. Parameters for Case 2 of Model 1.

\begin{tabular}{lllllllllllll}
$\boldsymbol{p}$ & $\boldsymbol{\beta}$ & $\boldsymbol{\theta}$ & $\boldsymbol{w}$ & $\boldsymbol{h}$ & $\boldsymbol{h}_{\mathbf{1}}$ & $\boldsymbol{h}_{\mathbf{2}}$ & $\boldsymbol{g}$ & $\boldsymbol{f}$ & $\boldsymbol{s}$ & $\boldsymbol{s}_{\mathbf{1}}$ & $\boldsymbol{s}_{\mathbf{2}}$ & $\boldsymbol{l}$ \\
\hline 3 & 0.6 & 0.1 & 2 & 0.6 & 0.3 & 0.1 & 0.1 & 0.5 & 20 & 20 & 20 & 150
\end{tabular}

As shown in Table 4, none of the parameters have any effect on $q_{12}^{*}$ except for $l$ and none of the parameters have any effect on $n_{12}^{*}$ except for $h_{1}, h_{2}$, and $l . p$ and $l$ have a positive correlation with $r_{12}^{*}$ and $T_{12}^{*}$. Except for $p$ and $l$, none of the parameters have any effect on $r_{12}^{*}$ and $T_{12}^{*} \cdot p, \beta, \theta, w$, and $l$ are positively related to $A P_{12}^{*}$ because the increment of revenue is larger than that of cost. $A P_{12}^{*}$ reduced as $h, h_{1}, h_{2}, g, c, s, s_{1}$, increased and $s_{2}$ caused the ordering cost, holding cost, and production cost to be increased.

6.3. Numerical example for Case 1 of Model 2 The parameters for Case 1 of Model 2 are listed in Table 5. The demand function is $d(p)=6000-500 p$. Running the Algorithm 2-1 provided in Subsection 5.2, we can obtain the following optimal results: $r_{21}^{*}=$ $0.042, T_{21}^{*}=0.042, n_{21}^{*}=1, q_{21}^{*}=104.88, A P_{21}^{*}=$ 4475.6. $r_{21}^{*}=T_{21}^{*}=0$ implies that the inventory of the retailer is zero. $n_{21}^{*}=1$ means that the inventory of the distribution center is zero.

We investigate the relationship of parameters and optimal solutions by sensitivity analysis. All parameters have four values. $p, \beta, \theta$, and $w$ reduce $10 \%$ at a time. $h, h_{1}, h_{2}, g, c, s, s_{1}$, and $s_{2}$ increase $10 \%$ at a time. $l$ increases $50 \%$ at a time. The results of sensitivity analysis are shown in Table 6 .

As we can see from Table 6, none of the parameters have any effect on $n_{21}^{*}$. $p$ has positive correlation with $r_{21}^{*}$ and $T_{21}^{*}$ has negative correlation with $q_{21}^{*} \cdot \beta$, $\theta, w, h, h_{1}, g, c$, and $l$ have no impact on $r_{21}^{*}, T_{21}^{*}$, and $q_{21}^{*}$. $h_{2}$ is negatively related to $r_{21}^{*}, T_{21}^{*}$, and $q_{21}^{*} . s, s_{1}$, and $s_{2}$ are positively related to $r_{21}^{*}, T_{21}^{*}$, and $q_{21}^{*} . p$ is positively related to $A P_{21}^{*}$ since sales revenue increases with increasing $p$. Because the inventory of retailer and distribution center is zero, $\beta, \theta, w, h, h_{1}, g$, and $l$ have no impact on $A P_{21}^{*}$. The reason for this is that the holding cost and ordering cost increase with increasing $h_{2}, c, s, s_{1}$, and $s_{2}$, which have a negative effect on $A P_{21}^{*}$.

\subsection{Numerical example for Case 2 of Model 2}

The parameters for Case 2 of Model 2 are given in

Table 7 . The demand function is $d(p)=5000-250 p$.

We carry out the Algorithm 2-2 presented in Subsection 5.2 and get the following optimal results:

$$
\begin{aligned}
& r_{22}^{*}=0, \quad T_{22}^{*}=0.0535, \quad n_{22}^{*}=5, \\
& q_{22}^{*}=150, \quad A P_{22}^{*}=23070.25
\end{aligned}
$$

$r_{22}^{*}=0$ means that there is no shortage. $q_{22}^{*}=150$ indicates the space of shelf has been used up.

\begin{tabular}{|c|c|c|c|c|c|c|}
\hline Parameter & Value & $n_{12}^{*}$ & $r_{12}^{*}$ & $T_{12}^{*}$ & $q_{12}^{*}$ & $A P_{12}^{*}$ \\
\hline \multirow[t]{4}{*}{$p$} & 3 & 8 & 0.0186 & 0.0186 & 150 & 18518.27 \\
\hline & 3.3 & 8 & 0.0190 & 0.0190 & 150 & 20536.73 \\
\hline & 3.6 & 8 & 0.0193 & 0.0193 & 150 & 22465.2 \\
\hline & 3.9 & 8 & 0.0197 & 0.0197 & 150 & 24303.66 \\
\hline \multirow[t]{4}{*}{$\beta$} & 0.6 & 8 & 0.0186 & 0.0186 & 150 & 18518.27 \\
\hline & 0.66 & 8 & 0.0186 & 0.0186 & 150 & 18528.73 \\
\hline & 0.72 & 8 & 0.0186 & 0.0186 & 150 & 18539.19 \\
\hline & 0.78 & 8 & 0.0186 & 0.0186 & 150 & 18549.64 \\
\hline \multirow[t]{4}{*}{$\theta$} & 0.1 & 8 & 0.0186 & 0.0186 & 150 & 18518.27 \\
\hline & 0.11 & 8 & 0.0186 & 0.0186 & 150 & 18519.19 \\
\hline & 0.12 & 8 & 0.0186 & 0.0186 & 150 & 18520.11 \\
\hline & 0.13 & 8 & 0.0186 & 0.0186 & 150 & 18521.03 \\
\hline \multirow[t]{4}{*}{$w$} & 2 & 8 & 0.0186 & 0.0186 & 150 & 18518.27 \\
\hline & 2.2 & 8 & 0.0186 & 0.0186 & 150 & 18519.76 \\
\hline & 2.4 & 8 & 0.0186 & 0.0186 & 150 & 18521.26 \\
\hline & 2.6 & 8 & 0.0186 & 0.0186 & 150 & 18522.76 \\
\hline \multirow[t]{4}{*}{$h$} & 0.6 & 8 & 0.0186 & 0.0186 & 150 & 18518.27 \\
\hline & 0.54 & 8 & 0.0186 & 0.0186 & 150 & 18522.76 \\
\hline & 0.48 & 8 & 0.0186 & 0.0186 & 150 & 18527.25 \\
\hline & 0.42 & 8 & 0.0186 & 0.0186 & 150 & 18531.74 \\
\hline \multirow[t]{4}{*}{$h_{1}$} & 0.3 & 8 & 0.0186 & 0.0186 & 150 & 18518.27 \\
\hline & 0.27 & 9 & 0.0186 & 0.0186 & 150 & 18536.09 \\
\hline & 0.24 & 9 & 0.0186 & 0.0186 & 150 & 18554.09 \\
\hline & 0.21 & 10 & 0.0186 & 0.0186 & 150 & 18572.7 \\
\hline \multirow[t]{4}{*}{$h_{2}$} & 0.1 & 8 & 0.0186 & 0.0186 & 150 & 18518.27 \\
\hline & 0.09 & 9 & 0.0186 & 0.0186 & 150 & 18524.84 \\
\hline & 0.08 & 9 & 0.0186 & 0.0186 & 150 & 18531.59 \\
\hline & 0.07 & 9 & 0.0186 & 0.0186 & 150 & 18538.34 \\
\hline \multirow[t]{4}{*}{$g$} & 0.1 & 8 & 0.0186 & 0.0186 & 150 & 18518.27 \\
\hline & 0.09 & 8 & 0.0186 & 0.0186 & 150 & 18518.34 \\
\hline & 0.08 & 8 & 0.0186 & 0.0186 & 150 & 18518.42 \\
\hline & 0.07 & 8 & 0.0186 & 0.0186 & 150 & 18518.49 \\
\hline \multirow[t]{4}{*}{$c$} & 0.5 & 8 & 0.0186 & 0.0186 & 150 & 18518.27 \\
\hline & 0.45 & 8 & 0.0186 & 0.0186 & 150 & 18920.89 \\
\hline & 0.4 & 8 & 0.0186 & 0.0186 & 150 & 19323.5 \\
\hline & 0.35 & 8 & 0.0186 & 0.0186 & 150 & 19726.12 \\
\hline \multirow[t]{4}{*}{$s$} & 20 & 8 & 0.0186 & 0.0186 & 150 & 18518.27 \\
\hline & 18 & 8 & 0.0186 & 0.0186 & 150 & 18625.63 \\
\hline & 16 & 8 & 0.0186 & 0.0186 & 150 & 18733 \\
\hline & 14 & 8 & 0.0186 & 0.0186 & 150 & 18840.36 \\
\hline \multirow[t]{4}{*}{$s_{1}$} & 20 & 8 & 0.0186 & 0.0186 & 150 & 18518.27 \\
\hline & 18 & 8 & 0.0186 & 0.0186 & 150 & 18531.69 \\
\hline & 16 & 8 & 0.0186 & 0.0186 & 150 & 18545.11 \\
\hline & 14 & 8 & 0.0186 & 0.0186 & 150 & 18558.53 \\
\hline \multirow[t]{4}{*}{$s_{2}$} & 20 & 8 & 0.0186 & 0.0186 & 150 & 18518.27 \\
\hline & 18 & 8 & 0.0186 & 0.0186 & 150 & 18531.69 \\
\hline & 16 & 8 & 0.0186 & 0.0186 & 150 & 18545.11 \\
\hline & 14 & 8 & 0.0186 & 0.0186 & 150 & 18558.53 \\
\hline \multirow[t]{4}{*}{$l$} & 150 & 8 & 0.0186 & 0.0186 & 150 & 18518.27 \\
\hline & 200 & 6 & 0.0248 & 0.0248 & 200 & 18817.73 \\
\hline & 250 & 5 & 0.0309 & 0.0309 & 250 & 19011.27 \\
\hline & 300 & 4 & 0.0370 & 0.0370 & 300 & 19149.87 \\
\hline
\end{tabular}

Table 4. Sensitivity analysis for Case 2 of Model 1. 
Table 5. Parameters for Case 1 of Model 2.

\begin{tabular}{ccccccccccccc}
\hline $\boldsymbol{p}$ & $\boldsymbol{\beta}$ & $\boldsymbol{\theta}$ & $\boldsymbol{w}$ & $\boldsymbol{h}$ & $\boldsymbol{h}_{\mathbf{1}}$ & $\boldsymbol{h}_{\mathbf{2}}$ & $\boldsymbol{g}$ & $\boldsymbol{f}$ & $\boldsymbol{s}$ & $\boldsymbol{s}_{\mathbf{1}}$ & $\boldsymbol{s}_{\mathbf{2}}$ & $\boldsymbol{l}$ \\
\hline 7 & 0.6 & 0.1 & 2 & 2 & 1 & 5 & 6 & 5 & 5 & 3 & 3 & 150 \\
\hline
\end{tabular}

Table 6. Sensitivity analysis for Case 2 of Model 1.

\begin{tabular}{|c|c|c|c|c|c|c|}
\hline Parameter & Value & $n_{21}^{*}$ & $r_{21}^{*}$ & $T_{21}^{*}$ & $q_{21}^{*}$ & $A P_{21}^{*}$ \\
\hline \multirow{4}{*}{$p$} & 7 & 1 & 0.0420 & 0.0420 & 104.88 & 4475.60 \\
\hline & 0.63 & 1 & 0.0393 & 0.0393 & 111.98 & 3145.09 \\
\hline & 0.56 & 1 & 0.0371 & 0.0371 & 118.66 & 1326.70 \\
\hline & 0.49 & 1 & 0.0352 & 0.0352 & 124.98 & -979.90 \\
\hline \multirow[t]{4}{*}{$\beta$} & 0.6 & 1 & 0.0420 & 0.0420 & 104.88 & 4475.60 \\
\hline & 0.54 & 1 & 0.0420 & 0.0420 & 104.88 & 4475.60 \\
\hline & 0.48 & 1 & 0.0420 & 0.0420 & 104.88 & 4475.60 \\
\hline & 0.42 & 1 & 0.0420 & 0.0420 & 104.88 & 4475.60 \\
\hline \multirow[t]{4}{*}{$\theta$} & 0.1 & 1 & 0.0420 & 0.0420 & 104.88 & 4475.60 \\
\hline & 0.09 & 1 & 0.0420 & 0.0420 & 104.88 & 4475.60 \\
\hline & 0.08 & 1 & 0.0420 & 0.0420 & 104.88 & 4475.60 \\
\hline & 0.07 & 1 & 0.0420 & 0.0420 & 104.88 & 4475.60 \\
\hline \multirow[t]{4}{*}{$w$} & 2 & 1 & 0.0420 & 0.0420 & 104.88 & 4475.60 \\
\hline & 1.8 & 1 & 0.0420 & 0.0420 & 104.88 & 4475.60 \\
\hline & 1.6 & 1 & 0.0420 & 0.0420 & 104.88 & 4475.60 \\
\hline & 1.4 & 1 & 0.0420 & 0.0420 & 104.88 & 4475.60 \\
\hline \multirow[t]{4}{*}{$h$} & 2 & 1 & 0.0420 & 0.0420 & 104.88 & 4475.60 \\
\hline & 2.2 & 1 & 0.0420 & 0.0420 & 104.88 & 4475.60 \\
\hline & 2.4 & 1 & 0.0420 & 0.0420 & 104.88 & 4475.60 \\
\hline & 2.6 & 1 & 0.0420 & 0.0420 & 104.88 & 4475.60 \\
\hline \multirow[t]{4}{*}{$h_{1}$} & 1 & 1 & 0.0420 & 0.0420 & 104.88 & 4475.60 \\
\hline & 1.1 & 1 & 0.0420 & 0.0420 & 104.88 & 4475.60 \\
\hline & 1.2 & 1 & 0.0420 & 0.0420 & 104.88 & 4475.60 \\
\hline & 1.3 & 1 & 0.0420 & 0.0420 & 104.88 & 4475.60 \\
\hline \multirow[t]{4}{*}{$h_{2}$} & 5 & 1 & 0.0420 & 0.0420 & 104.88 & 4475.60 \\
\hline & 5.5 & 1 & 0.0400 & 0.0400 & 100.00 & 4450.00 \\
\hline & 6 & 1 & 0.0383 & 0.0383 & 95.74 & 4425.54 \\
\hline & 6.5 & 1 & 0.0368 & 0.0368 & 91.99 & 4402.09 \\
\hline \multirow[t]{4}{*}{$g$} & 6 & 1 & 0.0420 & 0.0420 & 104.88 & 4475.60 \\
\hline & 6.6 & 1 & 0.0420 & 0.0420 & 104.88 & 4475.60 \\
\hline & 7.2 & 1 & 0.0420 & 0.0420 & 104.88 & 4475.60 \\
\hline & 7.8 & 1 & 0.0420 & 0.0420 & 104.88 & 4475.60 \\
\hline \multirow[t]{4}{*}{$c$} & 5 & 1 & 0.0420 & 0.0420 & 104.88 & 4475.60 \\
\hline & 5.5 & 1 & 0.0420 & 0.0420 & 104.88 & 3225.60 \\
\hline & 6 & 1 & 0.0420 & 0.0420 & 104.88 & 1975.60 \\
\hline & 6.5 & 1 & 0.0420 & 0.0420 & 104.88 & 725.60 \\
\hline \multirow[t]{4}{*}{$s$} & 5 & 1 & 0.0420 & 0.0420 & 104.88 & 4475.60 \\
\hline & 5.5 & 1 & 0.0429 & 0.0429 & 107.24 & 4463.81 \\
\hline & 6 & 1 & 0.0438 & 0.0438 & 109.54 & 4452.28 \\
\hline & 6.5 & 1 & 0.0447 & 0.0447 & 111.80 & 4440.98 \\
\hline \multirow[t]{4}{*}{$s_{1}$} & 3 & 1 & 0.0420 & 0.0420 & 104.88 & 4475.60 \\
\hline & 3.3 & 1 & 0.0425 & 0.0425 & 106.30 & 4468.49 \\
\hline & 3.6 & 1 & 0.0431 & 0.0431 & 107.70 & 4461.48 \\
\hline & 3.9 & 1 & 0.0436 & 0.0436 & 109.09 & 4454.56 \\
\hline \multirow[t]{4}{*}{$s_{2}$} & 3 & 1 & 0.0420 & 0.0420 & 104.88 & 4475.60 \\
\hline & 3.3 & 1 & 0.0425 & 0.0425 & 106.30 & 4468.49 \\
\hline & 3.6 & 1 & 0.0431 & 0.0431 & 107.70 & 4461.48 \\
\hline & 3.9 & 1 & 0.0436 & 0.0436 & 109.09 & 4454.56 \\
\hline \multirow[t]{4}{*}{$l$} & 150 & 1 & 0.0420 & 0.0420 & 104.88 & 4475.60 \\
\hline & 200 & 1 & 0.0420 & 0.0420 & 104.88 & 4475.60 \\
\hline & 250 & 1 & 0.0420 & 0.0420 & 104.88 & 4475.60 \\
\hline & 300 & 1 & 0.0420 & 0.0420 & 104.88 & 4475.60 \\
\hline
\end{tabular}

Table 7. Parameters for Case 2 of Model 2.

\begin{tabular}{ccccccccccccc}
\hline $\boldsymbol{p}$ & $\boldsymbol{\beta}$ & $\boldsymbol{\theta}$ & $\boldsymbol{w}$ & $\boldsymbol{h}$ & $\boldsymbol{h}_{\mathbf{1}}$ & $\boldsymbol{h}_{\mathbf{2}}$ & $\boldsymbol{g}$ & $\boldsymbol{f}$ & $\boldsymbol{s}$ & $\boldsymbol{s}_{\mathbf{1}}$ & $\boldsymbol{s}_{\mathbf{2}}$ & $\boldsymbol{l}$ \\
\hline 9 & 0.6 & 0.1 & 2 & 0.6 & 0.3 & 0.1 & 0.1 & 0.5 & 20 & 20 & 20 & 150 \\
\hline
\end{tabular}

We carry out the sensitivity analysis to investigate the impact of parameters on optimal solutions. Similar to Case 1 , all parameters have four values. $p, \beta, \theta$, and $w$ increase $10 \%$ at a time. $h, h_{1}, h_{2}, g, c, s, s_{1}$, and $s_{2}$ decrease $10 \%$ at a time. $l$ increases $50 \%$ at a time. The results are shown in Table 8 .

As we can see from Table 8, none of the parameters have any effect $r_{22}^{*}$. None of the parameters have any effect $n_{22}^{*}$ except for $p, h_{1}$, and $l$. And none of the parameters have any effect on $q_{22}^{*}$ except for $l . p$ and $l$ are positively related to $T_{22}^{*}$ and $\beta$ is negatively related to $T_{22}^{*}$. Other parameters do not affect $T_{22}^{*}$. According to the demand function, if the price equals 10, sales revenue reaches the maximum. Therefore, if the price is larger than 10 , price is negatively related to $A P_{22}^{*}$. If the price is smaller than 10 , price is positively related to $A P_{22}^{*}$. With the increase of $\beta, \theta, w$, and $l, A P_{22}^{*}$ also increased. The reason is that the added revenue is larger than the added cost. $A P_{22}^{*}$ decreased as $h, h_{1}, h_{2}, g, c, s, s_{1}$ increased and $s_{2}$ caused the ordering cost, holding cost, and production cost to be increased.

In summary, the impact of $p, \beta, \theta, l$, and $w$ on $A P_{i j}^{*}$ is complex. Whether the effect is positive or negative depends on the demand function and the values of these parameters. If the added revenue generated by increasing these parameters exceeds the added cost generated by increasing these parameters, the impact of these parameters is positive, and vice versa. Therefore, decision-makers should adjust the parameters according to the actual situation to maximize the average profits. The impact of $h, h_{1}, h_{2}, g, c, s, s_{1}$, and $s_{2}$ on $A P_{i j}^{*}$ is clear. If the value of these parameters increases, $A P_{i j}^{*}$ will be cut down. In other words, these parameters have a negative impact on $A P_{i j}^{*}$. Thus decision-makers should reduce these parameters as far as possible to cut down the cost. They could also choose direct sales to cut out the middleman and lower costs.

\section{Conclusions and future researches}

This paper proposes and optimizes two inventory models for perishable products with price and stockdependent demand in the supply chain. The supply chain is composed of one manufacturer, one distribution center, and one retailer. The objective is to maximize the average profit of the supply chain. The following conclusions can be made. In terms of model, 
Table 8. Sensitivity analysis for Case 2 of Model 2.

\begin{tabular}{|c|c|c|c|c|c|c|}
\hline Parameter & Value & $n_{22}^{*}$ & $r_{22}^{*}$ & $T_{22}^{*}$ & $q_{22}^{*}$ & $A P_{22}^{*}$ \\
\hline \multirow[t]{4}{*}{$p$} & 9 & 5 & 0 & 0.0535 & 150 & 23070.25 \\
\hline & 9.9 & 5 & 0 & 0.0582 & 150 & 23512.30 \\
\hline & 10.8 & 5 & 0 & 0.0638 & 150 & 23549.25 \\
\hline & 11.7 & 4 & 0 & 0.0705 & 150 & 23182.72 \\
\hline \multirow[t]{4}{*}{$\beta$} & 0.6 & 5 & 0 & 0.0535 & 150 & 23070.25 \\
\hline & 0.66 & 5 & 0 & 0.0534 & 150 & 23108.10 \\
\hline & 0.72 & 5 & 0 & 0.0533 & 150 & 23145.91 \\
\hline & 0.78 & 5 & 0 & 0.0532 & 150 & 23183.67 \\
\hline \multirow[t]{4}{*}{$\theta$} & 0.1 & 5 & 0 & 0.0535 & 150 & 23070.25 \\
\hline & 0.11 & 5 & 0 & 0.0535 & 150 & 23071.13 \\
\hline & 0.12 & 5 & 0 & 0.0535 & 150 & 23072.00 \\
\hline & 0.13 & 5 & 0 & 0.0535 & 150 & 23072.88 \\
\hline \multirow[t]{4}{*}{$w$} & 2 & 5 & 0 & 0.0535 & 150 & 23070.25 \\
\hline & 2.2 & 5 & 0 & 0.0535 & 150 & 23071.75 \\
\hline & 2.4 & 5 & 0 & 0.0535 & 150 & 23073.24 \\
\hline & 2.6 & 5 & 0 & 0.0535 & 150 & 23074.73 \\
\hline \multirow[t]{4}{*}{$h$} & 0.6 & 5 & 0 & 0.0535 & 150 & 23070.25 \\
\hline & 0.54 & 5 & 0 & 0.0535 & 150 & 23074.73 \\
\hline & 0.48 & 5 & 0 & 0.0535 & 150 & 23079.20 \\
\hline & 0.42 & 5 & 0 & 0.0535 & 150 & 23083.67 \\
\hline \multirow[t]{4}{*}{$h_{1}$} & 0.3 & 5 & 0 & 0.0535 & 150 & 23070.25 \\
\hline & 0.27 & 5 & 0 & 0.0535 & 150 & 23079.25 \\
\hline & 0.24 & 5 & 0 & 0.0535 & 150 & 23088.25 \\
\hline & 0.21 & 6 & 0 & 0.0535 & 150 & 23098.91 \\
\hline \multirow[t]{4}{*}{$h_{2}$} & 0.1 & 5 & 0 & 0.0535 & 150 & 23070.25 \\
\hline & 0.09 & 5 & 0 & 0.0535 & 150 & 23074.00 \\
\hline & 0.08 & 5 & 0 & 0.0535 & 150 & 23077.75 \\
\hline & 0.07 & 5 & 0 & 0.0535 & 150 & 23081.50 \\
\hline \multirow[t]{4}{*}{$g$} & 0.1 & 5 & 0 & 0.0535 & 150 & 23070.25 \\
\hline & 0.09 & 5 & 0 & 0.0535 & 150 & 23070.33 \\
\hline & 0.08 & 5 & 0 & 0.0535 & 150 & 23070.40 \\
\hline & 0.07 & 5 & 0 & 0.0535 & 150 & 23070.48 \\
\hline \multirow[t]{4}{*}{$c$} & 0.5 & 5 & 0 & 0.0535 & 150 & 23070.25 \\
\hline & 0.45 & 5 & 0 & 0.0535 & 150 & 23210.36 \\
\hline & 0.4 & 5 & 0 & 0.0535 & 150 & 23350.47 \\
\hline & 0.35 & 5 & 0 & 0.0535 & 150 & 23490.58 \\
\hline \multirow[t]{4}{*}{$s$} & 20 & 5 & 0 & 0.0535 & 150 & 23070.25 \\
\hline & 18 & 5 & 0 & 0.0535 & 150 & 23107.62 \\
\hline & 16 & 5 & 0 & 0.0535 & 150 & 23144.98 \\
\hline & 14 & 5 & 0 & 0.0535 & 150 & 23182.34 \\
\hline \multirow[t]{4}{*}{$s_{1}$} & 20 & 5 & 0 & 0.0535 & 150 & 23070.25 \\
\hline & 18 & 5 & 0 & 0.0535 & 150 & 23077.73 \\
\hline & 16 & 5 & 0 & 0.0535 & 150 & 23085.20 \\
\hline & 14 & 5 & 0 & 0.0535 & 150 & 23092.67 \\
\hline \multirow[t]{4}{*}{$s_{2}$} & 20 & 5 & 0 & 0.0535 & 150 & 23070.25 \\
\hline & 18 & 5 & 0 & 0.0535 & 150 & 23077.73 \\
\hline & 16 & 5 & 0 & 0.0535 & 150 & 23085.20 \\
\hline & 14 & 5 & 0 & 0.0535 & 150 & 23092.67 \\
\hline \multirow[t]{4}{*}{$l$} & 150 & 5 & 0 & 0.0535 & 150 & 23070.25 \\
\hline & 200 & 4 & 0 & 0.0709 & 200 & 23282.25 \\
\hline & 250 & 3 & 0 & 0.0881 & 250 & 23458.32 \\
\hline & 300 & 3 & 0 & 0.1051 & 300 & 23610.39 \\
\hline
\end{tabular}

eight propositions are put forward and proved for two models. These models could be reduced to at least four simplified models if one of the parameters is set to zero. Through sensitivity analysis, parameters that have positive and negative correlations with average profits are found. Decision-makers can adjust these parameters to achieve more profits. Taking direct sales is also a good way to increase profits. In terms of method, algorithms for two cases of two models are developed, and the effectiveness of these algorithms is demonstrated by computational experiments.

This paper provides an interesting topic for the optimization problem of inventory. However, there is some improvable room to extend this study. For example, we can study this problem under the assumption that the shortage is partial backlogging. Other demand-affecting elements, such as inflation and quantity discount, could be included in these models. Multi-objective and multi-product inventory optimization problems under uncertain environment could be considered. In addition, the joint optimization problem of location-inventory-routing is a promising research field.

\section{Acknowledge}

This work is supported by Society and Science Planning Projects in Jiangxi [grant number 19GL31], the Education Department of Jiangxi Province [grant number GJJ170956] and the National Natural Science Foundation of China [grant number 72061017, 71863021, 71462020, 71662019, 12071250]

\section{References}

1. Dai, Z., Aqlan, F., and Gao, K. "Optimizing multiechelon inventory with three types of demand in supply chain", Transp. Res. Pt. e-Logist. Transp. Rev., 107, pp. 141-177 (2017).

2. Gorji, M.H., Setak, M., and Karimi, H. "Optimizing inventory decisions in a two-level supply chain with order quantity constraints", Appl. Math. Model., 38(3), pp. 814-827 (2014).

3. Chu, Y., You, F., Wassick, J.M., et al. "Simulationbased optimization framework for multi-echelon inventory systems under uncertainty", Comput. Chem. Eng., 73, pp. 1-16 (2015).

4. Panda, D., Maiti, M.K., and Maiti, M. "Two warehouse inventory models for single vendor multiple retailers with price and stock dependent demand", Appl. Math. Model., 34(11), pp. 3571-3585 (2010).

5. Rad, M.A., Khoshalhan, F., and Glock, C.H. "Optimizing inventory and sales decisions in a two-stage supply chain with imperfect production and backorders", Comput. Ind. Eng., 74, pp. 219-227 (2014).

6. Sadeghi, J., Mousavi, S. M., Niaki, S.T. A., et al. "Optimizing a bi-objective inventory model of a 
three-echelon supply chain using a tuned hybrid bat algorithm", Transp. Res. Pt. e-Logist. Transp. Rev., 70, pp. 274-292 (2014).

7. Alhaj, M.A., Svetinovic, D., and Diabat, A. "A carbon-sensitive two-echelon-inventory supply chain model with stochastic demand", Resour. Conserv. Recycl., 108, pp. 82-87 (2016).

8. Mousavi, S.M., Alikar, N., Niaki, S.T.A., et al. "Optimizing a location allocation-inventory problem in a two-echelon supply chain network: A modified fruit fly optimization algorithm", Comput. Ind. Eng., 87, pp. 543-560 (2015).

9. Sarakhsi, M.K., Ghomi, S.F., and Karimi, B. "Joint economic lot-sizing problem for a two-stage supply chain with price-sensitive demand", Sci. Iran., 23(3), pp. 1474-1487 (2016).

10. Taleizadeh, A.A., Satarian, F., and Jamili, A. "Optimal multi-discount selling prices schedule for deteriorating product", Sci. Iran., 22(6), pp. 2595-2603 (2015).

11. Alfares, H.K. and Ghaithan, A.M. "Inventory and pricing model with price-dependent demand, timevaryng holding cost, and quantity discounts", Comput. Ind. Eng., 94, pp. 170-177 (2016).

12. Azadeh, A., Elahi, S., Farahani, M.H., et al. "A genetic algorithm-Taguchi based approach to inventory routing problem of a single perishable product with transshipment", Comput. Ind. Eng., 104, pp. 124-133 (2017).

13. Vahdani, B., Niaki, S.T.A., and Aslanzade, S. "Production-inventory-routing coordination with capacity and time window constraints for perishable products: Heuristic and meta-heuristic algorithms", $J$. Clean Prod., 161, pp. 598-618 (2017).

14. Cheng, M., Zhang, B., and Wang, G. "Optimal policy for deteriorating items with trapezoidal type demand and partial backlogging", Appl. Math. Model, 35(7), pp. 3552-3560 (2011).

15. Guerrero, W.J., Yeung, T.G., and Guéret, C. "Jointoptimization of inventory policies on a multi-product multi-echelon pharmaceutical system with batching and ordering constraints", Eur. J. Oper. Res., 231(1), pp. 98-108 (2013).

16. Tsai, S.C. and Liu, C.H. "A simulation-based decision support system for a multi-echelon inventory problem with service level constraints", Comput. Oper. Res., 53, pp. 118-127 (2015).

17. Zhao, Y. and Zhao, X. "On human decision behavior in multi-echelon inventory management", Int. J. Prod. Econ., 161, pp. 116-128 (2015).

18. Fichtinger, J. and Yates, N. "A joint network design and multi-echelon inventory optimisation approach for supply chain segmentation", Int. J. Prod. Econ., 209, pp. 103-111 (2017).

19. Ross, A., Khajehnezhad, M., Otieno, W., et al. "Integrated location-inventory modelling under forward and reverse product flows in the used merchandise retail sector: A multi-echelon formulation", Eur. J. Oper. Res., 259(2), pp. 664-676 (2017).

20. Wang, Z., Cui, B., Feng, Q., et al. "An agent-based approach for resources' joint planning in a multi-echelon inventory system considering lateral transshipment", Comput. Ind. Eng., 138, pp. 1-12 (2019).

21. Shen, H., Tian, T., and Zhu, H. "A two-echelon inventory system with a minimum order quantity requirement", Sustainability, 11(18), pp. 1-22 (2019).

22. Duan, Q. and Liao, T.W. "A new age-based replenishment policy for supply chain inventory optimization of highly perishable products", Int. J. Prod. Econ., 145(2), pp. 658-671 (2013).

23. Haijema, R. "Optimal ordering, issuance and disposal policies for inventory management of perishable products", Int. J. Prod. Econ., 157, pp. 158-169 (2014).

24. Coelho, L.C. and Laporte, G. "Optimal joint replenishment, delivery and inventory management policies for perishable products", Comput. Oper. Res., 47, pp. 42-52 (2014).

25. Liu, H., Zhang, J., Zhou, C., et al. "Optimal purchase and inventory retrieval policies for perishable seasonal agricultural products", Omega-Int. J. Manage. Sci., 79, pp. 133-145 (2018).

26. Kaasgari, M.A., Imani, D.M., and Mahmoodjanloo, M. "Optimizing a vendor managed inventory (VMI) supply chain for perishable products by considering discount: Two calibrated meta-heuristic algorithms", Comput. Ind. Eng., 103, pp. 227-241 (2017).

27. Jaggi, C.K., Cárdenas-Barrón, L.E., Tiwari, S., et al. "Two-warehouse inventory model for deteriorating items with imperfect quality under the conditions of permissible delay in payments", Sci. Iran., 24(1), pp. 390-412 (2017).

28. Teimoury, E. and Kazemi, S.M.M. "An integrated pricing and inventory model for deteriorating products in a two stage supply chain under replacement and shortage", Sci. Iran., 24(1), pp. 342-354 (2017).

29. Sarkar, B. and Sarkar, S. "An improved inventory model with partial backlogging, time varying deterioration and stock-dependent demand", Econ. Model., 30, pp. 924-932 (2013).

30. Panda, S., Saha, S., and Goyal, S.K. "Dilemma of rented warehouse and shelf for inventory systems with displayed stock level dependent demand", Econ. Model., 32, pp. 452-462 (2013).

31. Ghiami, Y., Williams, T., and Wu, Y. "A two-echelon inventory model for a deteriorating item with stockdependent demand, partial backlogging and capacity constraints", Eur. J. Oper. Res., 231(3), pp. 587-597 (2013).

32. Yang, C.T. "An inventory model with both stockdependent demand rate and stock-dependent holding cost rate", Int. J. Prod. Econ., 155, pp. 214-221 (2014). 
33. Bhunia, A.K., Shaikh, A.A., Dhaka, V., et al. "An application of genetic algorithm and PSO in an inventory model for single deteriorating item with variable demand dependent on marketing strategy and displayed stock level", Sci. Iran., 25(3), pp. 1641-1655 (2018).

34. Lim, S. "A note on a robust inventory model with stock-dependent demand", J. Oper. Res. Soc., 70(5), pp. 851-866 (2019).

35. Maihami, R. and Kamalabadi, I.N. "Joint pricing and inventory control for non-instantaneous deteriorating items with partial backlogging and time and price dependent demand", Int. J. Prod. Econ., 136(1), pp. 116-122 (2012).

36. Banerjee, S. and Agrawal, S. "Inventory model for deteriorating items with freshness and price dependent demand: optimal discounting and ordering policies", Appl. Math. Model., 52, pp. 53-64 (2017).

37. Jadidi, O., Jaber, M.Y., and Zolfaghari, S. "Joint pricing and inventory problem with price dependent stochastic demand and price discounts", Comput. Ind. Eng., 114, pp. 45-53 (2017).

38. San-José, L.A., Sicilia, J., and Alcaide-Lépez-dePablo, D. "An inventory system with demand dependent on both time and price assuming backlogged shortages", Eur. J. Oper. Res., 270(3), pp. 889-897 (2018).

39. Johari, M., Hosseini-Motlagh, S.M., Nematollahi, M., et al. "Bi-level credit period coordination for periodic review inventory system with price-credit dependent demand under time value of money", Transp. Res. Pt. e-Logist. Transp. Rev., 114, pp. 270-291 (2018).

40. Mishra, U., Wu, J.Z., and Tseng, M.L. "Effects of a hybrid-price-stock dependent demand on the optimal solutions of a deteriorating inventory system and trade credit policy on re-manufactured product", J. Clean Prod., 241, pp. 1-15 (2019).

41. Chen, L., Chen, X., Keblis, M.F., et al. "Optimal pricing and replenishment policy for deteriorating inventory under stock-level-dependent, time-varying and price-dependent demand", Comput. Ind. Eng., 135, pp. 1294-1299 (2019).

42. Pervin, M., Roy, S.K., and Weber, G.W. "Multi-item deteriorating two-echelon inventory model with priceand stock-dependent demand: A trade-credit policy", J. Ind. Manag. Optim., 15(3), pp. 1345-1373 (2019).

\section{Appendix A}

\section{The proof of Proposition 1}

For a given $n_{11}^{*}$ :

$$
p \beta+w \theta-h-\theta g-c(\beta+\theta) \leq 0 .
$$

$\frac{\partial A P_{11}\left(n_{11}^{*}, r_{11}, T_{11}\right)}{\partial r_{11}}<0$ if $r_{11}>0$ and $\frac{\partial A P_{11}\left(n_{11}^{*}, r_{11}, T_{11}\right)}{\partial r_{11}}>$ 0 if $r_{11}^{\partial r_{11}}<0$. Hence, $A P_{11}\left(n_{11}^{*}, r_{11}, T_{11}\right)^{\partial r_{11}}$ takes the maximum value when $r_{11}^{*}=0$. Plugging $r_{11}^{*}=0$ and Eq. (6) into Eq. (12), $A P_{11}$ can be written as:

$$
\begin{gathered}
A P_{11}\left(n_{11}^{*}, 0, T_{11}\right)=p d(p)-\frac{s}{T_{11}}-\frac{s_{1}}{n_{11}^{*} T_{11}} \\
-\frac{h_{1}\left(n_{11}^{*}-1\right) d(p) T_{11}}{2}-\frac{n_{11}^{*} d(p) T_{11} h_{2}}{2} \\
-\frac{s_{2}}{n_{11}^{*} T_{11}}-c d(p) .
\end{gathered}
$$

The first and second order derivatives of $A P_{11}$ with respect to $T_{11}$ are:

$$
\begin{aligned}
& \frac{\partial A P_{11}\left(n_{11}^{*}, 0, T_{11}\right)}{\partial T_{11}}= \frac{s}{T_{11}{ }^{2}}+\frac{s_{1}}{n_{11}^{*} T_{11}{ }^{2}} \\
&-\frac{h_{1}\left(n_{11}^{*}-1\right) d\left(p_{11}\right)}{2}-\frac{n_{11}^{*} d\left(p_{11}\right) h_{2}}{2}+\frac{s_{2}}{n_{11}^{*} T_{11}{ }^{2}}, \\
& \frac{\partial^{2} A P_{11}\left(n_{11}^{*}, 0, T_{11}\right)}{\partial T_{11}{ }^{2}}=-\frac{2 s}{T_{11}{ }^{3}}-\frac{2 s_{1}}{n_{11}^{*} T_{11}{ }^{3}} \\
&-\frac{2 s_{2}}{n_{11}^{*} T_{11}{ }^{3}}<0 .
\end{aligned}
$$

Eq. (A.3) implies $A P_{11}\left(n_{11}^{*}, 0, T_{11}\right)$ is strictly concave. Therefore, there is at most one solution $T_{11}^{*}$ to maximize $A P_{11}\left(n_{11}^{*}, 0, T_{11}\right)$ for a given $n_{11}^{*}$. $T_{11}^{*}$ could be solved by Eq. (A.2) $=0$. Plugging $T_{11}^{*}$ and $r_{11}^{*}$ into Eq. (7), we can obtain $q_{11}^{*}$. As $r_{11}^{*}=0$, the space limitation of the shelf is satisfied. The proof is completed.

\section{Appendix B}

The proof of Proposition 2

For a given $n_{121}^{*}$ :

$$
\frac{p \beta+w \theta-h-\theta g-c(\beta+\theta)}{T_{121}(\beta+\theta)}-\frac{h_{1}\left(n_{121}^{*}-1\right)+n_{121}^{*} h_{2}}{2}<0,
$$

$\frac{\partial A P_{121}\left(n_{121}^{*}, r_{121}, T_{121}\right)}{\partial r_{121}}<0$ if $r_{121}>0$ and $\frac{\partial A P_{121}\left(n_{121}^{*}, r_{121}, T_{121}\right)}{\partial r_{121}}>0$ if $r_{121}<0$. Hence, $A P_{121}\left(n_{121}^{*}, r_{121}, T_{121}\right)$ takes the maximum value when $r_{121}^{*}=0$. Plugging $r_{121}^{*}=0$ and Eq. (6) into Eq. (12), $A P_{121}$ can be written as:

$$
\begin{aligned}
A P_{121}( & \left.n_{121}^{*}, 0, T_{121}\right)=p d(p)-\frac{s}{T_{121}}-\frac{s_{1}}{n_{121} T_{121}} \\
- & \frac{h_{1}\left(n_{121}^{*}-1\right) d(p) T_{121}}{2}-\frac{n_{121}^{*} d(p) T_{121} h_{2}}{2} \\
- & \frac{s_{2}}{n_{121}^{*} T_{121}}-c d(p) .
\end{aligned}
$$


The first and second-order derivatives of $A P_{121}$ with respect to $T_{121}$ are:

$$
\begin{gathered}
\frac{\partial A P_{121}\left(n_{121}^{*}, 0, T_{121}\right)}{\partial T_{121}}=\frac{s}{T_{121}{ }^{2}}+\frac{s_{1}}{n_{121}^{*} T_{121}{ }^{2}} \\
-\frac{h_{1}\left(n_{121}^{*}-1\right) d(p)}{2}-\frac{n_{121}^{*} d(p) h_{2}}{2} \\
+\frac{s_{2}}{n_{121}^{*} T_{121}{ }^{2}}, \\
\frac{\partial^{2} A P_{121}\left(n_{121}^{*}, 0, T_{121}\right)}{\partial T_{121}{ }^{2}}=-\frac{2 s}{T_{121}{ }^{3}}-\frac{2 s_{1}}{n_{121}^{*} T_{121}{ }^{3}} \\
-\frac{2 s_{2}}{n_{121}^{*} T_{121}{ }^{3}}<0 .
\end{gathered}
$$

Eq. (B.3) implies $A P_{121}\left(n_{121}^{*}, 0, T_{121}\right)$ is strictly concave. Therefore, there is at most one solution $T_{121}^{*}$ to maximize $A P_{121}\left(n_{121}^{*}, 0, T_{121}\right)$ for a given $n_{121}^{*}$. $T_{121}^{*}$ could be obtained by solving Eq. (B.2) $=0$. Plugging $T_{121}^{*}$ and $r_{121}^{*}$ into Eq. (7), we can obtain $q_{121}^{*}$. As $r_{121}^{*}=0$, the space limitation of the shelf is satisfied.

In brief, for a given $n_{121}^{*}$, If:

$$
\begin{aligned}
& \frac{p \beta+w \theta-h-\theta g-c(\beta+\theta)}{T_{121}^{*}(\beta+\theta)} \\
&-\frac{h_{1}\left(n_{121}^{*}-1\right)+n_{121}^{*} h_{2}}{2}<0,
\end{aligned}
$$

we can calculate $A P_{121}^{*}\left(n_{121}^{*} 0, T_{121}^{*}\right)$ by plugging $n_{121}^{*}$ and $T_{121}^{*}$ into Eq. (12) otherwise $\left(n_{121}^{*}, 0, T_{121}^{*}\right)$ is not a feasible solution. The proof is completed.

\section{Appendix $\mathrm{C}$}

\section{The proof of proposition 3}

For a given $n_{122}^{*}$, if:

$$
\begin{aligned}
& \frac{p \beta+w \theta-h-\theta g-c(\beta+\theta)}{T_{122}(\beta+\theta)}-\frac{h_{1}\left(n_{122}^{*}-1\right)+n_{122}^{*} h_{2}}{2}>0, \\
& \frac{\partial A P_{122}\left(n_{122}^{*}, r_{122}, T_{122}\right)}{\partial r_{122}} \geq 0 .
\end{aligned}
$$

This means $A P_{122}\left(n_{122}^{*}, r_{122}, T_{122}\right)$ is a non-decreasing function. Therefore, $A P_{122}\left(n_{122}^{*}, r_{122}, T_{122}\right)$ reaches the maximum value when $r_{122}^{*}=T_{122}$.

Plugging Eq. (6) into Eq. (12) and replacing $r_{122}$ by $T_{122}$, we obtain the following equation:

$$
\begin{aligned}
A P_{122} & \left(n_{122}^{*}, T_{122}, T_{122}\right)=\frac{d(p)\left(e^{(\beta+\theta) T_{122}}-1\right)}{\beta+\theta} Z \\
& -\frac{s}{T_{122}}-\frac{s_{1}}{n_{122}^{*} T_{122}}-\frac{s_{2}}{n_{122}^{*} T_{122}}+p(d(p) \\
- & \left.\frac{d(p) \beta}{\beta+\theta}\right)-\frac{w d(p) \theta}{\beta+\theta}+\frac{h d(p)}{(\beta+\theta)}+\frac{d(p) \theta g}{(\beta+\theta)}
\end{aligned}
$$

$$
\begin{aligned}
Z= & \left(\frac{p \beta+w \theta-h-\theta g-c(\beta+\theta)}{T_{122}(\beta+\theta)}\right. \\
& \left.-\frac{h_{1}\left(n_{122}^{*}-1\right)+n_{122}^{*} h_{2}}{2}\right) .
\end{aligned}
$$

Taking the first-order derivative of Eq. (C.1) with respect to $T_{122}$, we obtain:

$$
\begin{gathered}
\frac{\partial A P_{122}\left(n_{122}^{*}, T_{122}, T_{122}\right)}{\partial T_{122}}=d(p) e^{(\beta+\theta) T_{122}} \\
Z+\frac{s}{T_{122}^{2}}+\frac{s_{1}}{n_{122}^{*} T_{122}^{2}}+\frac{s_{2}}{n_{122}^{*} T_{122}^{2}} .
\end{gathered}
$$

As $Z>0$,we have:

$$
\frac{\partial A P_{122}\left(n_{122}^{*}, T_{122}, T_{122}\right)}{\partial T_{122}}>0 .
$$

Hence, $A P_{122}\left(n_{122}^{*}, T_{122}, T_{122}\right)$ is an increasing function. $T_{122}$ should take the maximum value without exceeding the space limitation of the shelf. We replace $r_{122}$ by $T_{122}$ and let $q_{122}=l$ for Eq. (6). The following equation can be obtained:

$$
l=\frac{d(p)}{\beta+\theta}\left(e^{(\beta+\theta) T_{122}}-1\right) .
$$

Solving Eq. (C.3), we get $T_{122}$ by:

$$
T_{122}^{*}=\ln \left(\frac{l(\beta+\theta)}{d(p)}+1\right) /(\beta+\theta) .
$$

In brief, for a given $n_{122}^{*}$, if:

$$
\begin{array}{r}
\frac{p \beta+w \theta-h-\theta g-c(\beta+\theta)}{T_{122}^{*}(\beta+\theta)} \\
-\frac{h_{1}\left(n_{122}^{*}-1\right)+n_{122}^{*} h_{2}}{2}>0,
\end{array}
$$

we can calculate $A P_{122}^{*}\left(n_{122}^{*}, T_{122}^{*}, T_{122}^{*}\right)$ by plugging $n_{122}^{*}$ and $T_{122}^{*}$ into Eq. (12), otherwise $\left(n_{122}^{*}, T_{122}^{*}, T_{122}^{*}\right)$ is not a feasible solution. The proof is completed.

\section{Appendix D}

\section{The proof of Proposition 4}

We take the second-order derivative of Eq. (12) with respect to $r_{123}$, therefore, we can obtain the following equation:

$$
\begin{aligned}
& \frac{\partial^{2} A P_{123}\left(n_{123}, r_{123}, T_{123}\right)}{\partial r_{123}{ }^{2}}= \\
& (\beta+\theta) e^{(\beta+\theta) r_{123}} d(p) e^{(\beta+\theta) r_{123}} \\
& \left(\frac{p \beta+w \theta-h-\theta g-c(\beta+\theta)}{T_{123}(\beta+\theta)}\right. \\
& \left.-\frac{h_{1}\left(n_{123}-1\right)+n_{123} h_{2}}{2}\right) .
\end{aligned}
$$


Obviously,

$$
\frac{\partial^{2} A P_{123}\left(n_{123}, r_{123}, T_{123}\right)}{\partial r_{123}^{2}}=0,
$$

because:

$$
\frac{p \beta+w \theta-h-\theta g-c(\beta+\theta)}{T_{123}(\beta+\theta)}=\frac{h_{1}\left(n_{123}-1\right)+n_{123} h_{2}}{2} .
$$

Thus, the Hessian matrix $A P_{123}\left(n_{123}, r_{123}, T_{123}\right)$ satisfies the following equation:

$$
\begin{gathered}
\frac{\partial^{2} A P_{123}\left(n_{123}, r_{123}, T_{123}\right)}{\partial r_{123}{ }^{2}} \frac{\partial^{2} A P_{123}\left(n_{123}, r_{123}, T_{123}\right)}{\partial T_{123}{ }^{2}} \\
-\left(\frac{\partial^{2} A P_{123}\left(n_{123}, r_{123}, T_{123}\right)}{\partial T_{123} \partial r_{123}}\right)^{2} \leq 0
\end{gathered}
$$

Therefore, there is no optimal value for $A P_{123}\left(n_{123}\right.$, $\left.r_{123}, T_{123}\right)$. The proof is completed.

\section{Appendix E}

\section{The proof of proposition 5}

For a given $n_{21}^{*}$, if:

$$
\begin{aligned}
& p \beta+w \theta-h-\theta g-c(\beta+\theta) \leq 0, \\
& \frac{\partial A P_{21}\left(n_{21}^{*}, r_{21}, T_{21}\right)}{\partial r_{1}}<0 \text { when } r_{21}>T_{21} \text { and } \\
& \frac{\partial A P_{21}\left(n_{21}^{*}, r_{21}, T_{21}\right)}{\partial r_{21}}>0 \text { when } r_{21}<T_{21} .
\end{aligned}
$$

Hence, $A P_{21}\left(n_{21}^{*}, r_{21}, T_{21}\right)$ takes the maximum value when $r_{21}^{*}=T_{21}$. Plugging $r_{21}^{*}=T_{21}$ and Eq. (23) into Eq. (29), $A P_{21}$ can be obtained as follows:

$$
\begin{gathered}
A P_{21}\left(n_{21}^{*}, T_{21}, T_{21}\right)=p d(p)-\frac{s}{T_{21}} \\
-\frac{s_{1}}{n_{21}^{*} T_{21}}-\frac{h_{1}\left(n_{21}^{*}-1\right) d(p) T_{21}}{2} \\
-\frac{n_{21}^{*} d(p) T_{21} h_{2}}{2}-\frac{s_{2}}{n_{21}^{*} T_{21}}-c d(p) .
\end{gathered}
$$

The first and second-order derivatives of $A P_{21}$ with respect to $T_{21}$ are respectively:

$$
\begin{gathered}
\frac{\partial A P_{21}\left(n_{21}^{*}, T_{21}, T_{21}\right)}{\partial T_{21}}=\frac{s}{T_{21}{ }^{2}}+\frac{s_{1}}{n_{21}^{*} T_{21}{ }^{2}} \\
-\frac{h_{1}\left(n_{21}^{*}-1\right) d(p)}{2}-\frac{n_{21}^{*} d(p) h_{2}}{2}+\frac{s_{2}}{n_{21}^{*} T_{21}{ }^{2}}, \\
\frac{\partial^{2} A P_{21}\left(n_{21}^{*}, T_{21}, T_{21}\right)}{\partial T_{21}{ }^{2}}=-\frac{2 s}{T_{21}{ }^{3}}-\frac{2 s_{1}}{n_{21}^{*} T_{21}{ }^{3}} \\
-\frac{2 s_{2}}{n_{21}^{*} T_{21}{ }^{3}}<0 .
\end{gathered}
$$

Eq. (E.3) implies that there is at most one solution $T_{21}^{*}$ to maximize $A P_{21}\left(n_{21}^{*}, T_{21}, T_{21}\right)$ for a given $n_{21}^{*}$. $T_{21}^{*}$ could be solved by Eq. (E.2) $=0$. Plugging $T_{21}^{*}$ and $r_{21}^{*}$ into Eq. (24), we can obtain $q_{21}^{*}$. As $r_{21}^{*}=T_{21}$, the space limitation of the shelf is not violated. The proof is completed.

\section{Appendix F}

\section{The proof of Proposition 6}

For a given $n_{221}^{*}$, if

$$
\begin{aligned}
\frac{p \beta+w \theta-h-\theta g-c(\beta+\theta)}{T_{221}(\beta+\theta)} & \\
-\frac{h_{1}\left(n_{221}^{*}-1\right)+n_{221}^{*} h_{2}}{2} & <0, \\
\frac{\partial A P_{221}\left(n_{221}^{*}, r_{221}, T_{221}\right)}{\partial r_{221}} & <0
\end{aligned}
$$

if $r_{221}>T_{221}$ and $\frac{\partial A P_{221}\left(n_{21}^{*}, r_{221}, T_{221}\right)}{\partial r_{221}}>0$ if $r_{221}<$ $T_{221}$. Hence, $A P_{221}\left(n_{221}^{*}, r_{221}, T_{221}\right)$ takes the maximum value when $r_{221}^{*}=T_{221}$. Plugging $r_{221}^{*}=T_{221}$ and Eq. (24) into Eq. (29), $A P_{221}$ can be obtained as follows:

$$
\begin{aligned}
A P_{221}\left(n_{221}^{*}, T_{221}, T_{221}\right)=p d(p)-\frac{s}{T_{221}} & \\
- & \frac{s_{1}}{n_{221}^{*} T_{221}}-\frac{h_{1}\left(n_{221}^{*}-1\right) d(p) T_{221}}{2} \\
- & \frac{n_{221}^{*} d(p) T_{221} h_{2}}{2}-\frac{s_{2}}{n_{221}^{*} T_{221}}-c d(p) .
\end{aligned}
$$

The first and second-order derivatives of $A P_{221}$ with respect to $T_{221}$ are respectively:

$$
\begin{gathered}
\frac{\partial A P_{221}\left(n_{221}^{*}, T_{221}, T_{221}\right)}{\partial T_{221}}=\frac{s}{T_{221}{ }^{2}}+\frac{s_{1}}{n_{221}^{*} T_{221}{ }^{2}} \\
-\frac{h_{1}\left(n_{221}^{*}-1\right) d(p)}{2}-\frac{n_{221}^{*} d(p) h_{2}}{2}+\frac{s_{2}}{n_{221}^{*} T_{221}{ }^{2}}, \\
\frac{\partial^{2} A P_{221}\left(n_{221}^{*}, T_{221}, T_{221}\right)}{\partial T_{221}{ }^{2}}=-\frac{2 s}{T_{221}{ }^{3}}-\frac{2 s_{1}}{n_{221}^{*} T_{221}{ }^{3}} \\
-\frac{2 s_{2}}{n_{221}^{*} T_{221}{ }^{3}}<0 .
\end{gathered}
$$

Eq. (F.3) implies that there is at most one solution $T_{221}^{*}$ to maximize $A P_{221}\left(n_{221}^{*}, T_{221}, T_{221}\right)$ for a given $n_{221}^{*}$. $T_{221}^{*}$ could be solved by Eq. (F.2) $=0$. Plugging $T_{221}^{*}$ and $r_{221}^{*}$ into Eq. (24), we can obtain $q_{221}^{*}$. As $r_{221}^{*}=$ $T_{221}$, the space limitation of shelf is satisfied. In brief, for a given $n_{221}^{*}$, if: 


$$
\begin{array}{r}
\frac{p \beta+w \theta-h-\theta g-c(\beta+\theta)}{T_{221}^{*}(\beta+\theta)} \\
-\frac{h_{1}\left(n_{221}^{*}-1\right)+n_{221}^{*} h_{2}}{2}<0,
\end{array}
$$

we can calculate $A P_{221}^{*}\left(n_{221}^{*}, 0, T_{221}^{*}\right)$ by plugging $n_{221}^{*}$ and $T_{221}^{*}$ into Eq. (29). Otherwise, $\left(n_{221}^{*}, 0, T_{221}^{*}\right)$ is not a feasible solution. The proof is completed.

\section{Appendix G}

\section{The proof of Proposition 7}

For a given $n_{222}^{*}$, if:

$$
\frac{p \beta+w \theta-h-\theta g-c(\beta+\theta)}{T_{222}(\beta+\theta)}-\frac{h_{1}\left(n_{222}^{*}-1\right)+n_{222}^{*} h_{2}}{2}>0
$$

then $\frac{\partial A P_{222}\left(n_{222}^{*}, r_{222}, T_{222}\right)}{\partial r_{222}} \leq 0$. This means $A P_{222}$ $\left(n_{222}^{*}, r_{222}, T_{222}\right)$ is a non-increasing function. Therefore, $A P_{222}\left(n_{222}^{*}, r_{222}, T_{222}\right)$ reaches the maximum value when $r_{222}^{*}=0$.

Plugging Eq. (23) into Eq. (29) and replacing $r_{222}$ by 0 , the following equation can be obtained:

$$
\begin{aligned}
A P_{222} & \left(n_{222}^{*}, 0, T_{222}\right)=\frac{d(p)\left(e^{(\beta+\theta) T_{222}}-1\right)}{\beta+\theta} Y-\frac{s}{T_{222}} \\
& -\frac{s_{1}}{n_{222}^{*} T_{222}}-\frac{s_{2}}{n_{222}^{*} T_{222}}+p_{222}(d(p) \\
& \left.-\frac{d(p) \beta}{\beta+\theta}\right)-\frac{w d(p) \theta}{\beta+\theta}+\frac{h d(p)}{(\beta+\theta)}+\frac{d(p) \theta g}{(\beta+\theta)} . \quad \text { (G.1) }
\end{aligned}
$$

In Eq. (G.1):

$$
\begin{aligned}
Y= & \left(\frac{p \beta+w \theta-h-\theta g-c(\beta+\theta)}{T_{222}(\beta+\theta)}\right. \\
& \left.-\frac{h_{1}\left(n_{222}^{*}-1\right)+n_{222}^{*} h_{2}}{2}\right) .
\end{aligned}
$$

Taking the first-order derivative of Eq. (G.1) with respect to $T_{222}$, we obtain:

$$
\begin{gathered}
\frac{\partial A P_{222}\left(n_{222}^{*}, 0, T_{222}\right)}{\partial T_{222}}=d(p) e^{(\beta+\theta) T_{222}} Y+\frac{s}{T_{222}^{2}} \\
+\frac{s_{1}}{n_{222}^{*} T_{222}^{2}}+\frac{s_{2}}{n_{222}^{*} T_{222}^{2}} .
\end{gathered}
$$

As $Y>0$, we have $\frac{\partial A P_{222}\left(n_{222}^{*}, 0, T_{222}\right)}{\partial T_{222}}>0$. Hence, $A P_{222}\left(n_{222}^{*}, 0, T_{222}\right)$ is an increasing function. $T_{222}$ should take the maximum value without exceeding the space limitation of shelf. We replace $r_{222}$ by 0 and let $q_{222}=l$ for Eq. (23), as a result we obtain the following equation:

$$
l=\frac{d(p)}{\beta+\theta}\left(e^{(\beta+\theta) T_{222}}-1\right) .
$$

Solving Eq. (G.3), we get $T_{222}$ by:

$$
T_{222}^{*}=\ln \left(\frac{l(\beta+\theta)}{d\left(p_{222}\right)}+1\right) /(\beta+\theta) .
$$

In brief, for a given $n_{222}^{*}$, if:

$$
\begin{aligned}
& \frac{p \beta+w \theta-h-\theta g-c(\beta+\theta)}{T_{222}^{*}(\beta+\theta)} \\
& -\frac{h_{1}\left(n_{222}^{*}-1\right)+n_{222}^{*} h_{2}}{2}>0,
\end{aligned}
$$

we can calculate $A P_{222}^{*}\left(n_{222}^{*}, 0, T_{222}^{*}\right)$ by plugging $n_{222}^{*}$ and $T_{222}^{*}$ into Eq. (29), otherwise $\left(n_{222}^{*}, 0, T_{222}^{*}\right)$ is not a feasible solution. The proof is completed.

\section{Appendix $\mathbf{H}$}

\section{The proof of Proposition 8}

If:

$$
\frac{p \beta+w \theta-h-\theta g-c(\beta+\theta)}{T_{223}(\beta+\theta)}-\frac{h_{1}\left(n_{223}-1\right)+n_{223} h_{2}}{2}=0
$$

we take the second-order derivative of Eq. (29) with respect to $r_{223}$ :

$$
\begin{gathered}
\frac{\partial^{2} A P_{223}\left(n_{223}, r_{223}, T_{223}\right)}{\partial r_{223}{ }^{2}}= \\
(\beta+\theta) e^{(\beta+\theta)\left(T_{223}-r_{223}\right)} d(p) \\
\left(\frac{p \beta+w \theta-h-\theta g-c(\beta+\theta)}{T_{223}(\beta+\theta)}\right. \\
\left.-\frac{h_{1}\left(n_{223}-1\right)+n_{223} h_{2}}{2}\right) .
\end{gathered}
$$

Since:

$$
\begin{aligned}
& \frac{p \beta+w \theta-h-\theta g-c(\beta+\theta)}{T_{223}(\beta+\theta)}= \\
& \frac{h_{1}\left(n_{223}-1\right)+n_{223} h_{2}}{2}, \frac{\partial^{2} A P_{223}\left(n_{223}, r_{223}, T_{223}\right)}{\partial r_{223}{ }^{2}}=0 .
\end{aligned}
$$

Thus, the Hessian matrix of $A P_{223}\left(n_{223}, r_{223}\right.$, $T_{223}$ ) satisfies the following condition:

$$
\begin{gathered}
\frac{\partial^{2} A P_{223}\left(n_{223}, r_{223}, T_{223}\right)}{\partial r_{223}{ }^{2}} \cdot \frac{\partial^{2} A P_{223}\left(n_{223}, r_{223}, T_{223}\right)}{\partial T_{223}{ }^{2}} \\
-\left(\frac{\partial^{2} A P_{223}\left(n_{223}, r_{223}, T_{223}\right)}{\partial T_{223} \partial r_{223}}\right)^{2} \leq 0
\end{gathered}
$$

Therefore, there is no maximum value for $A P_{223}\left(n_{223}\right.$, $\left.r_{223}, T_{223}\right)$. The proof is completed. 


\section{Biographies}

Zhuo Dai is a Professor at the School of Ecommerce of Jiujiang University. He received his $\mathrm{PhD}$ degree from Xiamen University in 2011. His main research field is the modeling and optimization of logistics and supply chains. He has published several articles in journals indexed by SCI, such as Expert Systems with Applications, Transportation Research Part E, Computers \& Industrial Engineering.

Kuo Gao is a Professor at the School of Economics and Management of Jiujiang University. He received his PhD degree from Nanchang University in 2012. His main research field is management science and engineering. He has published several articles in journals indexed by SCI, such as Expert Systems with Applications, Transportation Research Part E.

Xiaoting Zheng is a Professor at the School of Ecommerce of Jiujiang University. He received his MS degree from South Central University for nationalities in 2001. His main research field is the modeling and optimization of logistics and supply chains. He has published a few articles in Computers \& Industrial Engineering. He has presided over several latitudinal projects. 